\title{
Are Khovanov-Rozansky polynomials consistent with evolution in the space of knots?
}

\section{A. Anokhina and A. Morozov}

\author{
Institute for Theoretical and Experimental Physics, \\ Bolshaya Cheryemushkinskaya 25, Moscow 117218, Russia \\ Institute for Information Transmission Problems, \\ Bolshoy Karetnyi 19-1, Moscow 127994, Russia \\ E-mail: anokhina@itep.ru, morozov@itep.ru
}

\begin{abstract}
R$-coloured knot polynomials for $m$-strand torus knots Torus ${ }_{[m, n]}$ are described by the Rosso-Jones formula, which is an example of evolution in $n$ with Lyapunov exponents, labelled by Young diagrams from $R^{\otimes m}$. This means that they satisfy a finitedifference equation (recursion) of finite degree. For the gauge group $\operatorname{SL}(N)$ only diagrams with no more than $N$ lines can contribute and the recursion degree is reduced. We claim that these properties (evolution/recursion and reduction) persist for Khovanov-Rozansky (KR) polynomials, obtained by additional factorization modulo $1+\mathbf{t}$, which is not yet adequately described in quantum field theory. Also preserved is some weakened version of differential expansion, which is responsible at least for a simple relation between reduced and unreduced Khovanov polynomials. However, in the KR case evolution is incompatible with the mirror symmetry under the change $n \longrightarrow-n$, what can signal about an ambiguity in the KR factorization even for torus knots.
\end{abstract}

Keywords: Chern-Simons Theories, Integrable Field Theories, Wilson, 't Hooft and Polyakov loops

ArXiv EPrint: 1802.09383 


\section{Contents}

1 Introduction 1

2 Recursion from Rosso-Jones formula for torus knots 3

$\begin{array}{lll}3 & \text { Recursion for torus superpolynomials } & 7\end{array}$

4 Recursion for torus Khovanov polynomials at $m=2 \quad 10$

5 Recursion for Khovanov $(N=2)$ polynomials at $m=3 \quad 13$

6 Recursion for Khovanov $(N=2)$ polynomials at $m=4 \quad 14$

$\begin{array}{lll}7 & \text { The case of } m=5 & 16\end{array}$

$\begin{array}{llr}8 & \text { Projective limit } & 19\end{array}$

9 Recursion for KR polynomials at $N=3$ and $m=4 \quad 20$

10 Remnant of differential expansion $\quad 22$

$\begin{array}{ll}11 \text { Conclusion } & 23\end{array}$

\section{Introduction}

Knot "polynomials" [1-8] are the vacuum expectation values of Wilson loops in $3 d$ ChernSimons theory [9-13], perhaps, refined [14-16]. Since the theory is topological they depend only on the linking properties of the loop $\mathcal{K}$ and do not change under smooth variations of its embedding into $3 d$ space $\mathcal{X}$. For simply connected $\mathcal{X}=R^{3}$ or $S^{3}$ they are rational functions (sometime, Laurent polynomials) of peculiar functions $q=\exp \left(\frac{2 \pi i}{g^{-2}+N}\right)$ and $A=q^{N}$ of the coupling constant $g$ and the rank $N-1$ of the gauge algebra $\mathcal{G}=\operatorname{sl}(N)$. The shape of the polynomials depend on $\mathcal{K}$ and representation $R$ of $\mathcal{G}$. The working definitions are in terms of the Reshetikhin-Turaev (RT) lattice theory [17-29] via Reidemeister-invariant convolutions of quantum $\mathcal{R}$-matrices at the nodes of the $2 d$ knot diagrams $\mathcal{D}$ and of Khovanov-Rozansky (KR) cohomological calculus [30]-[48] on the hypercubes, associated with $\mathcal{D}$. The central question in Chern-Simons theory is description of these rational functions on the (discrete) space of knots or knot diagrams - and it is a direct generalization of the similar question about $2 d$ conformal blocks [49-55], defined on the space of the 3-valent Feynman diagrams.

The first step in the story is of course the choice of parametrization of the space itself. Knot diagrams are often considered as glued from braids, and each $m$-strand braid has 
a natural decomposition into an ordered (noncommutative) product of the braid-group generators:

$$
\mathcal{B}\{n\}=\tau_{1}^{n_{1 \mid 1}} \ldots \tau_{m-1}^{n_{1 \mid m-1}} \tau_{1}^{n_{2 \mid 1}} \ldots \tau_{m-1}^{n_{2 \mid m-1}} \ldots
$$

The first task in the theory of knot polynomials is to describe their dependence on any of the integer-valued parameters $n$. This approach is called evolution method [56-60], because as a function of $n$ the polynomial, obtained by the modified RT method [26-29], is a sum of Lyapunov-like exponents:

$$
\mathcal{P}_{R}^{\mathcal{K}(n)}=\sum_{Q \in R^{\otimes m}} C_{R, Q} \cdot \lambda_{Q}^{n}
$$

over the Young diagrams $Q$ in the product $R^{\otimes m}$ with $n$-independent coefficients $C$. In other words, the polynomial satisfies a finite difference equation (recursion) in $n$,

$$
\left(\prod_{Q \in R^{\otimes m}} \hat{\nabla}_{\lambda_{Q}}^{(1)}\right) \mathcal{P}_{R}^{\mathcal{K}(n)}=0
$$

which can be integrated to a system

$$
\left(\prod_{\substack{Q \neq Q_{0} \\ Q \in R^{\otimes m}}} \hat{\nabla}_{\lambda_{Q} / \lambda_{Q_{0}}}^{(1)}\right)\left(\lambda_{Q_{0}}^{-n} \cdot \mathcal{P}_{R}^{\mathcal{K}(n)}\right)=C_{R, Q_{0}} \cdot \prod_{\substack{Q \neq Q_{0} \\ Q \in R^{\otimes m}}}\left(1-\frac{\lambda_{Q}}{\lambda_{Q_{0}}}\right)
$$

Here we use the finite difference operator

$$
\hat{\nabla}_{x}^{(m)} F_{n}=F_{n}-x \cdot F_{n-m}
$$

and do not divide by $1-x$ to simplify most formulas below. Also, we define the shift factor in $\nabla_{x}^{(m)}$ as $x$ rather than $x^{m}$, because this simplifies formulas for superpolynomials.

If some $p$ of the expansion coefficients $C$ are vanishing, the degree of the equation is actually reduced by $p$. In particular, this happens for $N<m|R|$, because all the diagrams $Q$ with more than $N$ lines do not contribute. There are also some "accidental" omissions for HOMFLY polynomials (like $C_{[1],[22]}$ in the case of torus $H^{[4, n]}$ ), but they are usually lifted after t-deformation to superpolynomials.

While evolution recursion is a direct consequence of RT approach, it is not yet so evident for KR method (see the first paper in [37-39] for a tedious derivation in the simplest example). Thus it is a meaningful question, if KR polynomials satisfy a recursion (1.4), and if its degree is further reduced at small $N$ ?

In the present paper we analyse the available list of KR polynomials for the simplest torus knots with $m=2,3,4,5$ in the fundamental representation $R=[1]=\square$ and observe that the answer is "yes": not only recursion is true, but its degree drops down at $N<m$, exactly as it does for the corresponding superpolynomials - where this followed from the super-Rosso-Jones formulas of [56].

If one assumes the recursion, then it provides an answer for infinitely many KR polynomials with all positive integer $n$ once the first few are known - what is a major achievement by itself, given the complexity of direct calculations in KR formalism. 
However, as already observed in [61], for some knot families the evolution formulas for superpolynomials provide a unified description only for positive values of the evolution parameter $n$, while fail to provide pure positive or pure negative polynomials for negative $n$. In this paper we find that the same happens to KR polynomials even for the torus family with more than two strands. In result, these functions of $n$ do not respect the mirror symmetries $n \longrightarrow-n$, at least in the usual way: while

$$
\mathcal{P}^{[m,-n]}(\mathbf{a}, \mathbf{q}, \mathbf{t})=\mathcal{P}^{[m, n]}\left(\mathbf{a}^{-1}, \mathbf{q}^{-1}, \mathbf{t}^{-1}\right)
$$

for torus superpolynomials from [56],

$$
\mathcal{K}^{[m,-n]}(N, \mathbf{q}, \mathbf{t}) \neq \mathcal{K}^{[m, n]}\left(N, \mathbf{q}^{-1}, \mathbf{t}^{-1}\right)
$$

for their KR counterparts, i.e. the left hand sides are understood as analytic continuations from positive $n$ to negative, provided by the evolution formulas. Despite mirror symmetry is not a true symmetry of knot theory, associated link diagrams are not Reidemeisterequivalent, this is still an unpleasant feature, signalling about some kind of non-analyticity of the KR reductions in the space of knots (or, perhaps, about non-adequate coordinatisation of this space by parameters like $n$ ). Of course, the problem (1.7) disappears in cases when KR reduction is trivial - exhausted by just a substitution $\mathbf{a}=\mathbf{q}^{N}$ in the superpolynomial, like it happens for reduced 2-strand $(m=2)$ torus polynomials at $N=2$. For the same reason mirror symmetry survives in the 3 -strand $(m=3)$ case for reduced torus polynomials at $N=2,3$ and for the unreduced ones at $N=2$.

As to another symmetry, acting on the family of knot polynomials, $[m, n] \leftrightarrow[n, m]$, which is topological and relates Reidemeister-equivalent link diagrams, it is respected by our evolution formulas: both are true,

$$
\mathcal{P}^{[m, n]}(\mathbf{a}, \mathbf{q}, \mathbf{t})=\mathcal{P}^{[n, m]}(\mathbf{a}, \mathbf{q}, \mathbf{t})
$$

and

$$
\mathcal{K}^{[m, n]}(N, \mathbf{q}, \mathbf{t})=\mathcal{K}^{[n, m]}(N, \mathbf{q}, \mathbf{t})
$$

\section{Recursion from Rosso-Jones formula for torus knots}

The simplest example of (1.2) appeared in the case of torus knots, when the knot diagram consists of a single $m$-strand braid (1.1) of a very special form: with all non-vanishing $n_{a \mid i}=1$,

$$
\mathcal{B}^{[m, n]}=\left(\tau_{1} \ldots \tau_{m-1}\right)^{n}
$$

One gets a knot when $n$ and $m$ are coprime, otherwise there is a link with $\operatorname{gcd}(m, n)$ components. For $m$-strand torus knot HOMFLY polynomial is given by the Rosso-Jones evolution formula $[56,62-66]$

$$
{ }^{F_{F}} H_{R}^{[m, n]}(A, q)=F_{R}^{n} \sum_{Q \in R^{\otimes m}} C_{Q} \cdot q^{-\frac{2 n}{m} \varkappa_{Q}} \cdot D_{Q}(A, q)
$$


where $D_{Q}=\operatorname{Schur}_{Q}^{*}:=\operatorname{Schur}_{Q}\left\{p_{k}=\frac{A^{k}-A^{-k}}{q^{k}-q^{-k}}\right\}$ are quantum dimensions of representations $Q$, given by the standard hook formula, the eigenvalues of cut-and-join (actually, Casimir) operator are $\varkappa_{Q}=\sum_{(i, j) \in Q}(i-j)$ and the integer-valued coefficients $C_{Q}$ are defined by the Adams rule

$$
\operatorname{Schur}_{R}\left\{p_{m k}\right\}=\sum_{Q \in R^{\otimes m}} C_{Q} \cdot \operatorname{Schur}_{Q}\left\{p_{k}\right\}
$$

Since the only dependence on $n$ is in the power of $q$, this HOMFLY satisfies a homogeneous finite-difference equation

$$
\prod_{Q \in R^{\otimes m}} \hat{\nabla}_{q^{-2 \varkappa} \cdot F_{R}^{m}}^{(m)} H_{R}^{[m, n]}=0
$$

where and the degree of the difference equation is naively the number of Young diagrams $Q \in R^{2 m}$. This fact is independent on the actual value of the coefficients $C_{Q}$, i.e. from the point of view of the equation, they are the free parameters, parametrising its solution. In this sense the equation does not provide too much information about the HOMFLY polynomials, still it reflects an important property — evolution rule — of the torus family with particular number $m$ of strands.

However, when some additional information is available, the degree of the equation can be decreased. For example, if the spectrum of Casimir eigenvalues $\varkappa_{Q}$ is degenerate, i.e. if two or more of them coincide, the corresponding $Q$ should appear only once in the product in (2.4). Such degeneracy is often preserved by the t-deformation.

If instead some of the coefficients $C$ are vanishing, the corresponding $\nabla$ can be omitted and the degree of the difference equation gets lower. The lift from HOMFLY to superpolynomials, however, usually lifts such degenerations: even if $C$ was vanishing for HOMFLY, its t-deformation does not, see examples below.

Another story is when vanishing are some dimensions $D_{Q}$. For $A=q^{N}$ contributing are only $Q$ with no more than $N$ columns, because otherwise $D_{Q}\left(q, q^{N}\right)=0$. The order of the difference equation is reduced accordingly. In particular, for $N=2 R$ and $Q$ are symmetric representations (two-column representations of $s l_{2}$ are equivalent to the singlecolumn ones). The main claim of the present paper will be that this reduction is preserved by the t-deformation - even after additional reduction from super- to KR polynomial. Still, in this section we continue with HOMFLY.

If one adjusts the framing "phase" $F_{R}$ so that it cancels the contribution from one of the $\varkappa_{Q}, F_{R}=q^{\frac{2}{m} \varkappa_{0}}$, then (2.4) can be promoted (integrated) to a more restrictive non-homogeneous equation with degree lower by one (i.e. one of the free parameters in its solution can be fixed):

$$
\prod_{\substack{Q \neq Q_{0} \\ Q \in R^{\otimes m}}} \hat{\nabla}_{q^{2\left(\varkappa_{Q_{0}}-\varkappa_{Q}\right)}}^{(m)}{ }^{F} H_{R}^{[m, n]}=C_{Q_{0}}(q, A) \cdot D_{Q_{0}}(q, A) \cdot \prod_{\substack{Q \neq Q_{0} \\ Q \in R^{\otimes m}}}\left(1-q^{2\left(\varkappa_{Q_{0}}-\varkappa_{Q}\right)}\right)
$$

These framings are usually different from topological

$$
\mathcal{F}_{R}^{n}=\left(A^{|R|} q^{-4 \varkappa_{R}}\right)^{n(m-1)}
$$


thus one needs additional care when considering Reidemeister-equivalent knot diagrams, like $[m, n]$ and $[m,-n]$ in the case of torus knots. Here and in what follows we denote polynomials in topological framing and associated quantities by calligraphic letters.

In this paper we

(a) consider only fundamental representation $R=[1]=\square$ and

(b) make the choice $Q_{0}=m R=[m]$ with $\varkappa_{[m]}=\frac{m(m-1)}{2}$, and omit indices $f$ in what follows.

Deviation from topological invariance in this case implies that

$$
H_{\square}^{[m,-n]}(A, q)=\left(\frac{\mathcal{F}_{\square}}{F_{\square}}\right)^{2 n} \cdot H_{\square}^{[m, n]}\left(A^{-1}, q\right)=\left(\frac{\mathcal{F}_{\square}}{F_{\square}}\right)^{2 n} \cdot H_{\square}^{[m, n]}\left(A^{-1}, q^{-1}\right)
$$

and

$$
{ }^{F} H_{\square}-\frac{F_{\square}}{\mathcal{F}_{\square}}:\{A q\}\{A / q\}
$$

The second equality in (2.7) follows from transformation law for HOMFLY polynomial from $R=\square$ to transposed $R^{t r}=\square=R$ and thus is an accidental feature of the fundamental representation. Relation (2.7) persists in the case of superpolynomials, but generically it does not survive in the case of their KR reductions.

At $m=2$ the fundamental HOMFLY in this framing is

$$
H_{\square}^{[2, n]}=\frac{\{A q\}\{A\}}{\{q\}\left\{q^{2}\right\}}-q^{2 n} \cdot \frac{\{A\}\{A / q\}}{\{q\}\left\{q^{2}\right\}}
$$

and satisfies

$$
\hat{\nabla}_{q^{4}}^{(2)} H_{\square}^{[2, n]}=\frac{\{A q\}\{A\}}{\{q\}\left\{q^{2}\right\}} \cdot\left(1-q^{4}\right)
$$

Associated Jones polynomial (i.e. HOMFLY at $A=q^{2}$ )

$$
J_{\square}^{[2, n]}=[3]-q^{2 n}
$$

satisfies the same

$$
\hat{\nabla}_{q^{4}}^{(2)} J_{\square}^{[2, n]}=[3] \cdot\left(1-q^{4}\right)
$$

Here and below we use the standard notation: $\{x\}=x-x^{-1}$ and $[k]=\frac{\left\{q^{x}\right\}}{\{q\}}$.

At $m=3$

$$
H_{\square}^{[3, n]}=\frac{\left\{A q^{2}\right\}\{A q\}\{A\}}{\{q\}\left\{q^{2}\right\}\left\{q^{3}\right\}}-q^{2 n} \cdot \frac{\{A q\}\{A\}\{A / q\}}{\{q\}^{2}\left\{q^{3}\right\}}+q^{4 n} \cdot \frac{\{A\}\{A / q\}\left\{A / q^{2}\right\}}{\{q\}\left\{q^{2}\right\}\left\{q^{3}\right\}}
$$

satisfies the second-order difference equation

$$
\hat{\nabla}_{q^{6}}^{(3)} \hat{\nabla}_{q^{12}}^{(3)} H_{\square}^{[3, n]}=\left(1-q^{6}\right)\left(1-q^{12}\right) \cdot \frac{\left\{A q^{2}\right\}\{A q\}\{A\}}{\{q\}\left\{q^{2}\right\}\left\{q^{3}\right\}}
$$

while associated Jones polynomial

$$
J_{\square}^{[3, n]}=[4]-[2] \cdot q^{2 n}
$$


satisfies just the first-order one:

$$
\hat{\nabla}_{q^{6}}^{(3)} J_{\square}^{[3, n]}=[4] \cdot\left(1-q^{6}\right)
$$

At $m=4$

$$
\begin{aligned}
H_{\square}^{[4, n]}= & \frac{\left\{A q^{3}\right\}\left\{A q^{2}\right\}\{A q\}\{A\}}{\{q\}\left\{q^{2}\right\}\left\{q^{3}\right\}\left\{q^{4}\right\}}-q^{2 n} \cdot \frac{\left\{A q^{2}\right\}\{A q\}\{A\}\{A / q\}}{\{q\}^{2}\left\{q^{2}\right\}\left\{q^{4}\right\}}+0 \cdot q^{3 n} \cdot \frac{\{A q\}\{A\}^{2}\{A / q\}}{\{q\}\left\{q^{2}\right\}^{2}\left\{q^{3}\right\}}+ \\
& +q^{4 n} \cdot \frac{\{A q\}\{A\}\{A / q\}\left\{A / q^{2}\right\}}{\{q\}^{2}\left\{q^{2}\right\}\left\{q^{4}\right\}}-q^{6 n} \cdot \frac{\{A\}\{A / q\}\left\{A / q^{2}\right\}\left\{A / q^{3}\right\}}{\{q\}\left\{q^{2}\right\}\left\{q^{3}\right\}\left\{q^{4}\right\}}
\end{aligned}
$$

we get the first degeneracy: $C_{[2,2]}=0$. Thus the equation is of the order three, $\#_{\text {Young diagrams }}-1-\#_{C=0}=5-1-1=3$ :

$$
\hat{\nabla}_{q^{8}}^{(4)} \hat{\nabla}_{q^{16}}^{(4)} \hat{\nabla}_{q^{24}}^{(4)} H_{\square}^{[4, n]}=\left(1-q^{8}\right)\left(1-q^{16}\right)\left(1-q^{24}\right) \cdot \frac{\left\{A q^{3}\right\}\left\{A q^{2}\right\}\{A q\}\{A\}}{\{q\}\left\{q^{2}\right\}\left\{q^{3}\right\}\left\{q^{4}\right\}}
$$

This time the degree of equation is further decreased at $N=3$ and $N=2$ :

$$
H_{\square}^{[4, n]}\left(A=q^{3}, q\right)=\frac{[5][6]}{[2]}-[5][3] \cdot q^{2 n}+[3] \cdot q^{4 n}
$$

satisfies the second-order

$$
\hat{\nabla}_{q^{8}} \hat{\nabla}_{q^{16}} H_{\square}^{[4, n]}\left(A=q^{3}, q\right)=\frac{[5][6]}{[2]} \cdot\left(1-q^{8}\right)\left(1-q^{16}\right)
$$

while Jones polynomial

$$
J_{\square}^{[4, n]}=H_{\square}^{[4, n]}\left(A=q^{2}, q\right)=[5]-[3] \cdot q^{2 n}
$$

satisfies the first-order

$$
\hat{\nabla}_{q^{8}} J_{\square}^{[4, n]}=[5] \cdot\left(1-q^{8}\right)
$$

With increasing strand number $m$ we get the following pattern:

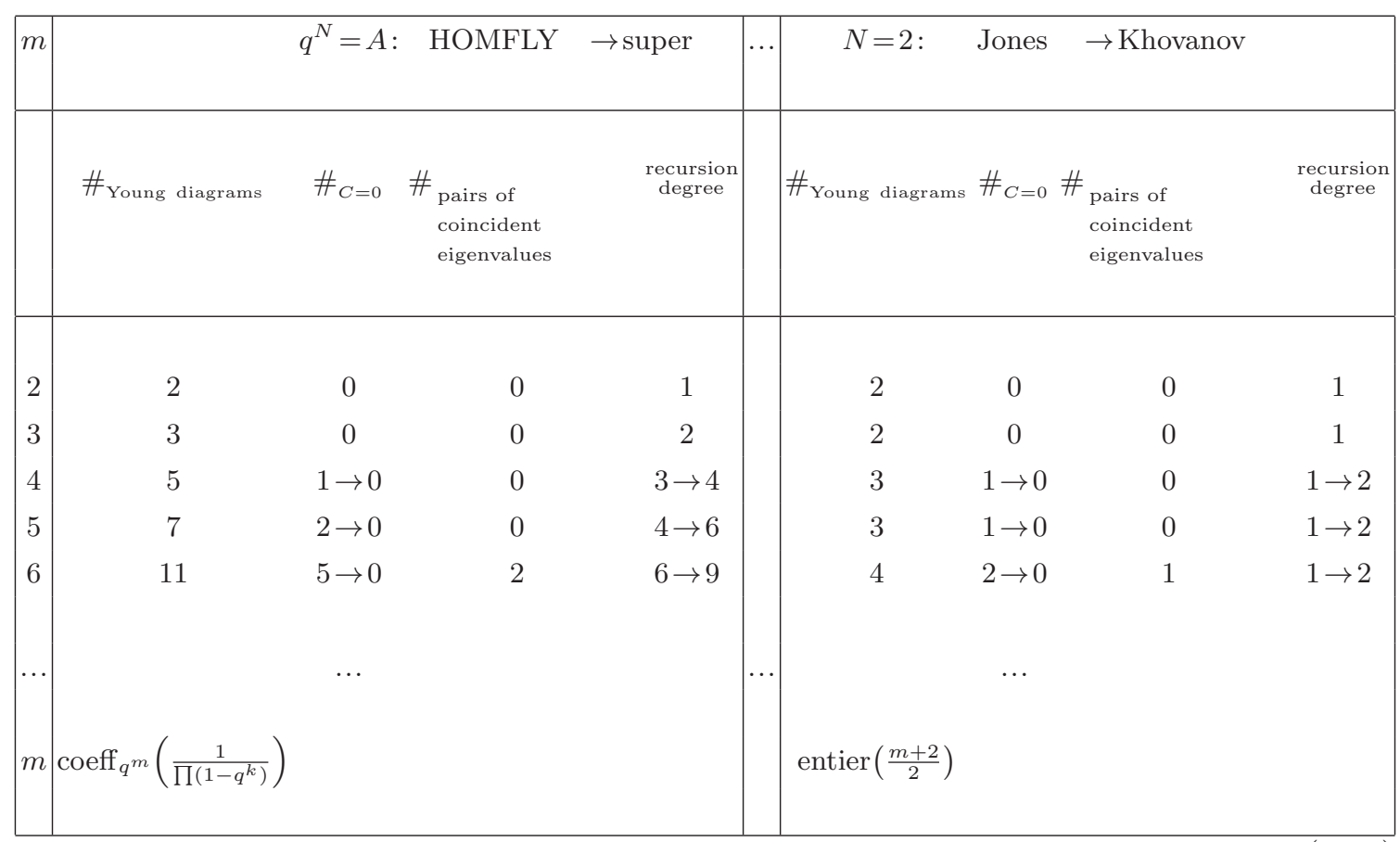


Arrows mark the changes which take place at the level of super- and Khovanov polynomials.

Reversing the logic, evolution recursion describes generic solutions of the homogeneous difference equation: from

$$
\hat{\nabla}_{x_{1}}^{(m)} \ldots \hat{\nabla}_{x_{p}}^{(m)} H_{n}=0
$$

it follows that

$$
H_{n}=\sum_{i=1}^{p} C_{i} \cdot x_{i}^{n / m}
$$

with $p$ arbitrary integration constants $C_{i}$. Each of these parameters appears in nonhomogeneous equation of degree $p-1$, obtained by integrating (2.24) w.r.t. one of the commuting difference operators $\hat{\Delta}_{x_{k}}$ :

$$
\left(\prod_{i \neq k}^{p} \hat{\nabla}_{x_{i}}^{(m)}\right) H_{n}=C_{k} \cdot x_{k}^{n / m} \cdot \prod_{i \neq k}^{p}\left(1-\frac{x_{i}}{x_{k}}\right)
$$

or

$$
\left(\prod_{i \neq k}^{p} \hat{\nabla}_{x_{i} / x_{k}}^{(m)}\right)\left(x_{k}^{-n / m} H_{n}\right)=C_{k} \cdot \prod_{i \neq k}^{p}\left(1-\frac{x_{i}}{x_{k}}\right)
$$

where the r.h.s. is independent of $n$.

Alternatively parameters $C_{k}$ can be defined from "initial conditions", i.e. the values of $H_{n}$ at some $p$ different values of the evolution parameter ("time") $n$. Used for this purpose below are the directly calculated KR polynomials for relatively small knots. Things would be greatly simplified if also their twins at negative values of $n$ could be used. However, the transformation $n \leftrightarrow-n$ acts badly on the evolution formulas for KR polynomials, and this way to extend the set of available initial conditions does not seem to work.

\section{Recursion for torus superpolynomials}

Superpolynomials depending on one extra deformation parameter $\mathbf{t} \neq-1$ could be defined from an analogue of the rule (2.3) with MacDonald instead of the Schur polynomials - and already this would make them depending on $\mathbf{q}$ and $\mathbf{t},-$ but actually there are additional correcting $c$-factors, discovered in [56] and better described in two different ways in [67] and [68-70].

Explicit expressions for the simplest reduced superpolynomials in topological framing are [56]:

$$
\begin{aligned}
\mathcal{P}_{\square}^{[2, n]}= & \left(\frac{A \cdot q}{t}\right)^{n} \cdot \frac{\{A q\} \cdot q^{-n}-\{A / t\} \cdot t^{n}}{\{q t\}} \\
\mathcal{P}_{\square}^{[3, n]}= & \left(\frac{A \cdot q}{t}\right)^{2 n} \\
& \cdot\left(\frac{\left\{A q^{2}\right\}\{A q\} \cdot q^{-2 n}}{\left\{q^{2} t\right\}\{q t\}}-\left(t^{ \pm 2}+1+q^{\mp 2}\right) \cdot \frac{\{A q\}\{A / t\} \cdot(t / q)^{2(n \mp 1) / 3}}{\left\{q^{2} t\right\}\left\{q t^{2}\right\}}+\frac{\{A / t\}\left\{A / t^{2}\right\} \cdot t^{2 n}}{\left\{q t^{2}\right\}\{q t\}}\right)
\end{aligned}
$$




$$
\begin{aligned}
\mathcal{P}_{\square}^{[4, n]}= & \left(\frac{A \cdot q}{t}\right)^{3 n} \cdot\left(\frac{\left\{A q^{3}\right\}\left\{A q^{2}\right\}\{A q\} \cdot q^{-3 n}}{\left\{q^{3} t\right\}\left\{q^{2} t\right\}\{q t\}}+\frac{\{t / q\} \cdot\{A q\}\{A q / t\}\{A / t\} \cdot(t / q)^{n}}{\left\{q^{2} t\right\}\left\{q t^{2}\right\}\{q t\}^{2}}-\frac{\{A / t\}\left\{A / t^{2}\right\}\left\{A / t^{3}\right\} \cdot t^{3 n}}{\left\{q t^{3}\right\}\left\{q t^{2}\right\}\{q t\}}\right. \\
& - \begin{cases}-\frac{\left(q t^{2}+q+q^{-1}+q^{-3}\right) \cdot\left\{A q^{2}\right\}\{A q\}\{A / t\} \cdot q^{-n} \cdot(t / q)^{(n-1) / 2}}{\left\{q^{3} t\right\}\left\{q^{2} t^{2}\right\}\{q t\}} \\
\left.+\frac{\left(t^{4} q^{-1}+t^{2} q^{-1}+q^{-1}+q^{-3}\right) \cdot\{A q\}\{A / t\}\left\{A / t^{2}\right\} \cdot t^{n} \cdot(t / q)^{(n-3) / 2}}{\left\{q^{2} t^{2}\right\}\left\{q t^{3}\right\}\{q t\}}\right) & n=1 \bmod m \\
-\frac{\left(q^{4} t^{-1}+q^{2} t^{-1}+t^{-1}+t^{-3}\right) \cdot\left\{A q^{2}\right\}\{A q\}\{A / t\} \cdot q^{-n} \cdot(t / q)}{\left\{q^{3} t\right\}\left\{q^{2} t^{2}\right\}\{q t\}} & \\
\left.+\frac{\left(t q^{2}+t+t^{-1}+t^{-3}\right) \cdot\{A q\}\{A / t\}\left\{A / t^{2}\right\} \cdot t^{n} \cdot(t / q)^{(n+1) / 2}}{\left\{q^{2} t^{2}\right\}\left\{q t^{3}\right\}\{q t\}}\right) & n=-1 \bmod m\end{cases}
\end{aligned}
$$

where $n= \pm 1 \bmod m$. Note that this parameter takes the opposite values for the knots $[m,-n]$ and $[m, n]$ in (1.6). Here we used our usual notation $\{x\}=x-x^{-1}$ and the standard MacDonald variables $A, q, t$, used in [56], are related to DGR variables of [35] by $A^{2}=-\mathbf{a}^{2} \mathbf{t}, q=-\mathbf{q} \mathbf{t}, t=\mathbf{q}$. Torus superpolynomials can be converted from Laurent to true superpolynomials by extracting a power of $A / t=\sqrt{-\mathbf{a}^{2} \mathbf{t} / \mathbf{q}^{2}} \longrightarrow \sqrt{-\mathbf{q}^{2 N-2} \mathbf{t}}$ : $\mathcal{P}_{[1]}^{[m, n]}=(A / t)^{(m-1)(n-1)} \cdot P_{[1]}^{[m, n]}=(A / t)^{(m-1)(n-1)} \cdot\left(1+O\left(A^{2}, t, q\right)\right)$ - note that $w=$ $(n-1)(m-1)$ appears here instead of the writhe number $n(m-1)$ in the exponents of the framing factor in (3.1). This explains the appearance of normalization factors in the following sections of this paper.

Superpolynomials are supposed to be positive Laurent polynomials of the DGR variables a, q, t, i.e., all the coefficients should be non-negative integers. However, this is not fully true for above expressions: with the change of $n$ they switch from pure positive to pure negative polynomials. Moreover, this is a typical anomaly: by insertion of additional overall factor of $(-)^{\frac{n-1}{m}}$ one could cure the problem for all positive values of $n$, but then all the polynomials with negative $n$ will be pure negative - violating also the invariance (1.6). Sometime this anomaly can cause serious problems for construction of superpolynomials [61], but for the torus knots per se this is a rather innocent detail, which, however, should be remembered and taken into account. It leads to a minor modification in the case of reduced super- and KR polynomials, while for the unreduced ones it is also minor, but can look somewhat unfamiliar, see below.

\begin{tabular}{|c|c|c|c|c|c|}
\hline$n$ & $n$ & \multicolumn{2}{|l|}{$\mathcal{P}_{\square}^{[n, m]}$} & & \\
\hline 2 & $2 k+p$ & $V_{r \mid 2}\left(A^{2} / t^{2}\right)^{n}$ & $+V_{r \mid 11}\left(A^{2} q^{2}\right)^{n}$ & & \\
\hline 3 & $3 k+p$ & $V_{r \mid 3}\left(A^{6} / t^{6}\right)^{n}$ & $+V_{r \mid 21}\left(A^{6}\right)^{n}$ & $+V_{r \mid 111}\left(A^{6} q^{6}\right)^{n}$ & \\
\hline 4 & $4 k+p$ & $V_{r \mid 4}\left(A^{12} t^{-12}\right)^{n}$ & $+V_{r \mid 31}\left(A^{12} q^{6} t^{-10}\right)^{n}+V_{r \mid 22}\left(A^{12} q^{8} t^{-8}\right)^{n}$ & $+V_{r \mid 211}\left(A^{12} q^{10} t^{-6}\right)^{n}$ & $+V_{r \mid 1111}\left(A^{12} q^{12}\right)^{n}$ \\
\hline & $N \geq$ & 1 & 2 & 3 & 4 \\
\hline
\end{tabular}

Expressions (3.1) for the superpolynomials have the form

The last line in this table is to remind that for $N \leq m$ only the eigenvalues from the first $N$ columns do contribute to the answer, while the coefficients $V$ of the remaining ones vanish 
in these cases. Such polynomials satisfy the recursion relations

$$
\begin{aligned}
& \nabla_{A^{2} q^{2}}^{(2)} \quad \nabla_{A^{2} / t^{2}}^{(2)} \quad P_{\square}^{[2, n]}=0 \\
& \nabla_{A^{6} q^{6}}^{(3)} \quad \nabla_{A^{6}}^{(3)} \quad \nabla_{A^{6} / t^{6}}^{(3)} \quad P_{\square}^{[3, n]}=0 \\
& \nabla_{A^{12} q^{12}}^{(4)} \nabla_{A^{12} q^{10} t^{-6}}^{(4)} \nabla_{A^{12} q^{8} t^{-8}}^{(4)} \nabla_{A^{12} q^{6} t^{-10}}^{(4)} \nabla_{A^{12} t^{-12}}^{(4)} P_{\square}^{[4, n]}=0 \\
& \underbrace{\underbrace{\underbrace{}_{N \geq 2}}_{N \geq 3}}_{N \geq 4}
\end{aligned}
$$

generated by the difference operators

$$
\hat{\nabla}_{\lambda}^{(m)} P_{\square}^{m, n} \equiv P_{\square}^{m, n}-\lambda \cdot P_{\square}^{m, n-m} .
$$

Since for $N \leq m$ the polynomials $P_{\square}^{n, m}$ in fact depend only on the first few eigenvalues, they actually satisfy the shortened recursions, which are generated by the difference operators, which survive in the last line in the table (3.3).

Equations (3.3) can be integrated to

$$
\begin{aligned}
\hat{\nabla}_{q^{2} t^{2}}^{(2)}\left((A / t)^{-n} \cdot \mathcal{P}_{\square}^{[2, n]}\right) & =\text { const }_{n} \\
\hat{\nabla}_{q^{4} t^{2}}^{(3)}\left((A / t)^{-2 n} \cdot \mathcal{P}_{\square}^{[3, n]}\right) & =\text { const }_{n} \bmod \left\{A / t^{2}\right\} \\
\hat{\nabla}_{q^{6} t^{2}}^{(4)} \hat{\nabla}_{q^{8} t^{4}}^{(4)}\left((A / t)^{-3 n} \cdot \mathcal{P}_{\square}^{[4, n]}\right) & =\text { const }_{n} \bmod \left\{A / t^{2}\right\},
\end{aligned}
$$

if we multiply each $\mathcal{P}_{\square}$ on the normalisation factor that makes the eigenvalue for $Q_{0}=[m]$ to be equal 1. Recursions for $m=3$ and $m=4$ here are the short ones, true for $A=t^{2}$, when $\left\{A / t^{2}\right\}=0$, i.e., they are valid for (super)Jones polynomials at $N=2$ : for generic $N$ the degrees of the equations would be 3 and 5 (or 4 for HOMFLY in the case of $m=4$, when representation [22] does not contribute). Note that for $m=4$ one does not expect difference equations with $\hat{\nabla}^{(m / 2)}$, because the coefficients in the superpolynomials depend on the residue $n \bmod m$.

Lyapunov exponents for the torus $n$-evolution are made from MacDonald split of the Casimir eigenvalues: for the Young diagram $Q=\left\{Q_{1} \geq Q_{2} \geq \ldots \geq Q_{l}>0\right\}$

$$
\lambda_{Q}^{n}=\left(\frac{A q}{t}\right)^{n(m-1)} \cdot\left(q^{-\nu\left(Q^{t r}\right)} \cdot t^{\nu(Q)}\right)^{2 n / m}=\left(-\mathbf{a}^{2} \mathbf{t}\right)^{n(m-1)} \cdot \mathbf{q}^{-\frac{2 n \varkappa(Q)}{m}} \cdot(-\mathbf{t})^{-\frac{2 n \nu\left(Q^{t r}\right)}{m}}
$$

where $\nu(Q)=\sum_{i=1}^{l}(i-1) Q_{i}$ and $\nu(Q)-\nu\left(Q^{t r}\right)=\varkappa(Q)=\sum_{(i, j) \in Q}(i-j)$. The spectra of the $m$-th powers $\left(\frac{\lambda_{Q}}{\lambda_{[m]}}\right)^{m}$, which enter the difference operators in the short recursion and are independent of $A$ and, consequently, on the details of the $N$-reduction, can be 
extracted from [56]:

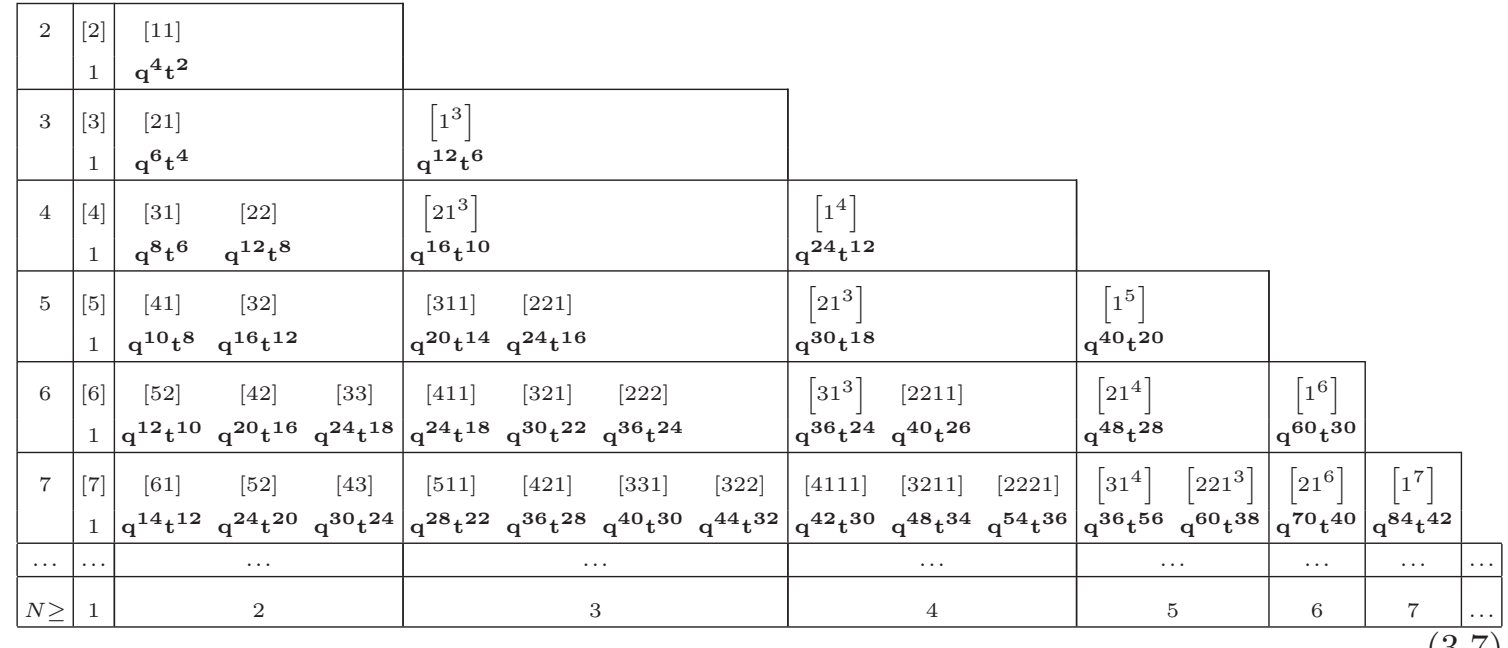

KR polynomials $K_{R}^{\mathcal{K}}(N, \mathbf{q}, \mathbf{t})$ are related to superpolynomial [34] by the DGR rule [35]: ${ }^{1}$

$$
K_{R}^{\mathcal{K}}(N, \mathbf{q}, \mathbf{t})=\left.P_{R}^{\mathcal{K}}(\mathbf{a}, \mathbf{q}, \mathbf{t}) \bmod \left\{A / t^{N}\right\}\right|_{\mathbf{a}=\mathbf{q}^{N}}
$$

The choice of the coefficient in front of the "differential"

$$
d_{N}=1+\frac{\mathbf{a}^{2} \mathbf{t}}{\mathbf{q}^{2 N}} \stackrel{\mathbf{a}=\mathbf{q}^{N}}{\longrightarrow} 1+\mathbf{t}
$$

is, however, not specified. In other words,

$$
K_{R}^{\mathcal{K}}(N, \mathbf{q}, \mathbf{t})=P_{R}^{\mathcal{K}}\left(\mathbf{a}=\mathbf{q}^{N}, \mathbf{q}, \mathbf{t}\right)-\underbrace{(1+\mathbf{t})}_{d_{N}\left(\mathbf{a}=\mathbf{q}^{N}, \mathbf{q}, \mathbf{t}\right)} \cdot \xi_{R}^{\mathcal{K}}(N, \mathbf{q}, \mathbf{t})
$$

and additional principles are needed to separate positive $\xi$ and positive $\mathcal{K}$, which also contains positive terms divisible by $1+\mathbf{t}$ - which could be, but are not moved to $\xi$. The search for these principles is a big challenge, but it is beyond the scope of the present text.

\section{Recursion for torus Khovanov polynomials at $m=2$}

In this case Khovanov polynomials are known from direct calculation at all odd $n$ [30, 32, $35-39]$ and they actually coincide with the result of the substitution of $N=2$ into

$$
\begin{aligned}
& \left(-\mathbf{q}^{\mathbf{2}(\mathbf{N}-\mathbf{1})} \mathbf{t}\right)^{-k} \cdot \mathcal{P}_{r}^{[2,2 k+1]}=\frac{A}{t} \cdot\left(\frac{M_{[2]}^{*}}{M_{[1]}^{*}}-\left(\mathbf{q}^{2} \mathbf{t}\right)^{2 k+1} \cdot \frac{\left\{\mathbf{q}^{2}\right\}}{\left\{\mathbf{q}^{\mathbf{2}} \mathbf{t}\right\}} \cdot \frac{M_{[11]}^{*}}{M_{[1]}^{*}}\right)= \\
& =\frac{1-\left(\mathbf{q}^{2} \mathbf{t}\right)^{2 k+2}}{1-\left(\mathbf{q}^{2} \mathbf{t}\right)^{2}}+\mathbf{q}^{2 N+2} \mathbf{t}^{3} \cdot \frac{1-\left(\mathbf{q}^{2} \mathbf{t}\right)^{2 k}}{1-\left(\mathbf{q}^{2} \mathbf{t}\right)^{2}}=1+\mathbf{q}^{\mathbf{4}} \mathbf{t}^{2}\left(1+\mathbf{q}^{2 N-2} \mathbf{t}\right) \cdot \frac{1-\left(\mathbf{q}^{2} \mathbf{t}\right)^{2 k}}{1-\left(\mathbf{q}^{\mathbf{2}} \mathbf{t}\right)^{2}}
\end{aligned}
$$

${ }^{1}$ The standard definition in the literature actually includes additional factor: $K_{R}^{\mathcal{K}}(N, \mathbf{q}, \mathbf{t})=$ $\left.(-\mathbf{t})^{-(m-1)(n-1) / 2} \cdot P_{R}^{\mathcal{K}}(\mathbf{a}, \mathbf{q}, \mathbf{t}) \bmod \left\{A / t^{N}\right\}\left(\mathbf{a}^{2} \mathbf{t}+\mathbf{q}^{2 N}\right)\right|_{\mathbf{a}=\mathbf{q}^{N}}$. We, however, omit it because this leads to significant simplifications in the logic, while it is always easy to restore the factor in the answers. Note that, like the superpolynomials (3.1) our $\mathcal{K}^{[m, n]}$ can be pure negative rather than pure positive. Also the reduced $N=2$ polynomials computed with the standard programs [33, 71] contain one more extra factor $\mathbf{q}^{-1}$, which we also omit. 
for reduced and

$$
\left(-\mathbf{q}^{\mathbf{2 ( N - 1 )}} \mathbf{t}\right)^{-k} \cdot \mathcal{P}_{u r}^{[2,2 k+1]}=[N]+\mathbf{q}^{3} \mathbf{t}^{2}\left(1+\mathbf{q}^{2 N} \mathbf{t}\right) \cdot \frac{1-\left(\mathbf{q}^{2} \mathbf{t}\right)^{2 k}}{1-\left(\mathbf{q}^{\mathbf{2}} \mathbf{t}\right)^{2}} \cdot[N-1]
$$

for unreduced superpolynomials. As explained in [40], relation between the two is implied by the differential expansion of the reduced fundamental superpotential:

$$
\mathcal{P}_{r}=1+\mathcal{F}\{A q\}\{A / t\}
$$

and for $N$-reduction $\{A / t\} \longrightarrow\left(1+\mathbf{q}^{\mathbf{2 N}-\mathbf{2}} \mathbf{t}\right)$. Naively unreduced polynomial is obtained by multiplication with the quantum number $[N]$. However, one can use the usual identification rule [40]

$$
\left(1+\mathbf{q}^{2} \mathbf{t}\right) \cdot[k] \cong \frac{1}{\mathbf{q}^{k-1}}\left(1+\mathbf{q}^{2 \mathbf{k}} \mathbf{t}\right)
$$

to substitute

$$
\begin{array}{ccc}
\multicolumn{4}{c}{\mathbf{q}^{N-2}\left(1+\mathbf{q}^{2} \mathbf{t}\right) \cdot[N][N-1]} & \\
\swarrow & \cong & \searrow \\
\left(1+\mathbf{q}^{2 \mathbf{N}-\mathbf{2}} \mathbf{t}\right) \cdot[N] & \cong & \frac{1}{\mathbf{q}} \cdot\left(1+\mathbf{q}^{2 \mathbf{N}} \mathbf{t}\right) \cdot[N-1]
\end{array}
$$

- and this is how (4.2) is obtained from (4.1).

Putting $N=2$, we obtain from (4.1)

$$
\mathcal{K}_{r}^{[2,2 k+1]}=\left(-\mathbf{q}^{2} \mathbf{t}\right)^{k} \cdot K_{r}^{[2,2 k+1]}=\left(-\mathbf{q}^{2} \mathbf{t}\right)^{k} \cdot\left(1+\mathbf{q}^{\mathbf{4}} \mathbf{t}^{2} \cdot \frac{1-\left(\mathbf{q}^{2} \mathbf{t}\right)^{2 k}}{1-\mathbf{q}^{\mathbf{2}} \mathbf{t}}\right)
$$

for reduced case and

$$
\mathcal{K}_{u r}^{[2,2 k+1]}=\left(-\mathbf{q}^{\mathbf{2}} \mathbf{t}\right)^{k} \cdot K_{u r}^{[2,2 k+1]}=\left(-\mathbf{q}^{2} \mathbf{t}\right)^{k} \cdot\left(\mathbf{q}+\mathbf{q}^{-1}+\mathbf{q}^{\mathbf{3}} \mathbf{t}^{\mathbf{2}}\left(1+\mathbf{q}^{4} \mathbf{t}\right) \cdot \frac{1-\left(\mathbf{q}^{2} \mathbf{t}\right)^{2 k}}{1-\left(\mathbf{q}^{\mathbf{2}} \mathbf{t}\right)^{2}}\right)
$$

from (4.2) for the unreduced one. Both of them satisfy non-homogeneous first-order equations:

$$
\begin{aligned}
& \nabla_{\mathbf{q}^{4} \mathbf{t}^{2}}^{(2)} K_{r}^{[2, n]}=K_{r}^{[2, n]}-\mathbf{q}^{\mathbf{4}} \mathbf{t}^{2} K_{r}^{[2, n-2]}=1+\mathbf{q}^{\mathbf{6}} \mathbf{t}^{\mathbf{3}} \\
& \nabla_{\mathbf{q}^{4} \mathbf{t}^{2}}^{(2)} K_{u r}^{[2, n]}=\mathbf{q}+\mathbf{q}^{-1}-\mathbf{q}^{\mathbf{5}} \mathbf{t}^{\mathbf{2}}+\mathbf{q}^{\mathbf{7}} \mathbf{t}^{\mathbf{3}}=\left(\mathbf{q}+\mathbf{q}^{-1}\right)\left(1+\mathbf{q}^{\mathbf{6}} \mathbf{t}^{\mathbf{3}}\right)-\underline{\mathbf{q}^{\mathbf{5}} \mathbf{t}^{\mathbf{2}}(1+\mathbf{t})}
\end{aligned}
$$

Note that these are exactly the same recursions as the one in the first line of (3.5): $q^{2} t^{2}=$ $\mathbf{q}^{4} \mathbf{t}^{2}$, only now we provided explicit expressions for the right hand sides. One can eliminate these right hand sides by applying one more difference operator, then the equation is the same for reduced and unreduced polynomials:

$$
\begin{aligned}
& \nabla_{1}^{(2)} \nabla_{\mathbf{q}^{4} \mathbf{t}^{2}}^{(2)} K_{\square}^{[2, n]}=K_{\square}^{[2, n]}-\left(1+\mathbf{q}^{4} \mathbf{t}^{2}\right) K_{\square}^{[2, n-2]}+\mathbf{q}^{\mathbf{4}} \mathbf{t}^{\mathbf{2}} K_{\square}^{[2, n-4]}=0 \\
& \uparrow \\
& \nabla_{-\mathbf{q}^{2} \mathbf{t}}^{(2)} \nabla_{-\mathbf{q}^{\mathbf{6}} \mathbf{t}^{\mathbf{3}}}^{(2)} \mathcal{K}_{\square}^{[2, n]}=\mathcal{K}_{\square}^{[2, n]}+\left(\mathbf{q}^{\mathbf{2}} \mathbf{t}+\mathbf{q}^{\mathbf{6}} \mathbf{t}^{\mathbf{3}}\right) \mathcal{K}_{\square}^{[2, n-2]}+\mathbf{q}^{\mathbf{8}} \mathbf{t}^{\mathbf{4}} \mathcal{K}_{\square}^{[2, n-4]}=0
\end{aligned}
$$


with

$$
\begin{aligned}
& A^{2} / t^{2}=-\mathbf{a}^{2} \mathbf{t} / \mathbf{q}^{2} \quad \stackrel{\mathbf{a}=\mathbf{q}^{2}}{\longrightarrow}-\mathbf{q}^{2} \mathbf{t} \\
& A^{2} q^{2}=-\mathbf{a}^{2} \mathbf{t}^{3} \mathbf{q}^{2} \quad \stackrel{\mathrm{a}=\mathbf{q}^{2}}{\longrightarrow}-\mathbf{q}^{\mathbf{6}} \mathbf{t}^{3}
\end{aligned}
$$

Invariance w.r.t. inversion of $n$ would state:

$$
\mathcal{K}_{r}^{[2,-n]}(\mathbf{q}, \mathbf{t})=\mathcal{K}_{r}^{[2, n]}\left(\frac{1}{\mathbf{q}}, \frac{1}{\mathbf{t}}\right) \quad \Longrightarrow \quad K_{r}^{[2,-n]}(\mathbf{q}, \mathbf{t})=-\mathbf{q}^{2} \mathbf{t} \cdot K_{r}^{[2, n]}\left(\frac{1}{\mathbf{q}}, \frac{1}{\mathbf{t}}\right)
$$

It is easy to check that the first recursion for $K_{r}$ in (4.8) is indeed invariant under this transformation, i.e. (4.11) is indeed true - what is not a surprise, because $K_{r}$ is obtained by a change of variables from the topologically invariant reduced superpolynomial (4.1). However, this is not the case for the unreduced $K_{u r}$ - like the unreduced superpolynomial (4.2), it changes non-trivially under the $n$-inversion (unless $\mathbf{t}=-1$ ), because of the underlined term in (4.8). The second branch differs from (4.7) by a slight modification of the first term:

$$
\tilde{K}_{u r}^{[2,2 k+1]}=-\mathbf{q} \mathbf{t}+\mathbf{q}^{-1}+\mathbf{q}^{3} \mathbf{t}^{2}\left(1+\mathbf{q}^{4} \mathbf{t}\right) \cdot \frac{1-\left(\mathbf{q}^{2} \mathbf{t}\right)^{2 k}}{1-\left(\mathbf{q}^{2} \mathbf{t}\right)^{2}}
$$

Instead of (4.11) the evolution formula for the unreduced Khovanov polynomial satisfies

$$
\mathcal{K}_{u r}^{[2,-n]}(\mathbf{q}, \mathbf{t})=\tilde{\mathcal{K}}_{u r}^{[2, n]}\left(\frac{1}{\mathbf{q}}, \frac{1}{\mathbf{t}}\right) \Longleftrightarrow K_{u r}^{[2,-n]}(\mathbf{q}, \mathbf{t})=-\mathbf{q}^{2} \mathbf{t} \cdot \tilde{K}_{u r}^{[2, n]}\left(\frac{1}{\mathbf{q}}, \frac{1}{\mathbf{t}}\right)
$$

We can now inverse the logic: begin from the recursion relation (3.5) and derive the evolution formula for arbitrary $K_{\square}^{[2, n]}$. Short and long recursions for $m=2$ mean that

$$
K_{\square}^{[2, n]}=\alpha^{\prime}+\beta^{\prime} \cdot \lambda^{2 n} \Longleftrightarrow \mathcal{K}^{[2, n]}=\alpha \cdot \lambda^{n}+\beta \cdot \lambda^{3 n}
$$

with $\lambda=\left(-\mathbf{q}^{2} \mathbf{t}\right)^{1 / 2}$ and some $n$-independent $\alpha$ and $\beta$. Note that at $N=2$ the two Lyapunov exponents differ by a factor of 3 , which is independent of parameters $\mathbf{q}$ and $\mathbf{t}$. To solve the recursion we need initial conditions and the knowledge of just two should be enough. It can seem that we always have these two: $n= \pm 1$ correspond to the unknot. The problem, however, is that we need (4.11) to use the both. If (4.11) is true - like it is for reduced KR polynomial, we immediately get:

$$
\begin{aligned}
& \alpha \cdot \lambda^{n}+\left.\beta \cdot \lambda^{3 n}\right|_{n= \pm 1}=\mathcal{K}_{r}^{\text {unknot }}=1 \Longrightarrow \\
& { }_{r} \mathcal{K}_{\square}^{[2, n]}=\left(-\mathbf{q}^{2} \mathbf{t}\right)^{\frac{n-1}{2}} \cdot\left(1+\mathbf{q}^{\mathbf{4}} \mathbf{t}^{2} \cdot \frac{1-\left(\mathbf{q}^{\mathbf{2}} \mathbf{t}\right)^{n-1}}{1-\mathbf{q}^{\mathbf{2}} \mathbf{t}}\right)=\left(-\mathbf{q}^{\mathbf{2}} \mathbf{t}\right)^{\frac{n+1}{2}} \cdot\left(1-\frac{1}{\mathbf{q}^{\mathbf{2}} \mathbf{t}} \cdot \frac{1-\left(\mathbf{q}^{2} \mathbf{t}\right)^{n+1}}{1-\mathbf{q}^{\mathbf{2}} \mathbf{t}}\right),
\end{aligned}
$$

i.e., reproduce (4.6). Note that the input as initial conditions was only the unknot (!) — this illustrates the usual power of evolution method. However, for unreduced KR polynomial we rather need (4.13), not (4.11):

$$
\begin{aligned}
& \alpha \cdot \lambda^{n}+\left.\beta \cdot \lambda^{3 n}\right|_{n= \pm 1}=\mathcal{K}_{u r}^{\text {unknot }}=\mathbf{q}+\left.(-\mathbf{t})^{\frac{n-1}{2}} \cdot \mathbf{q}^{-1}\right|_{n= \pm 1} \Longrightarrow \\
& \Longrightarrow{ }_{u r} \mathcal{K}_{\square}^{[2, n]}=\left(-\mathbf{q}^{2} \mathbf{t}\right)^{\frac{n-1}{2}} \cdot\left(\mathbf{q}+\mathbf{q}^{-1}+\mathbf{q}^{\mathbf{3}} \mathbf{t}^{\mathbf{2}}\left(1+\mathbf{q}^{\mathbf{4}} \mathbf{t}\right) \cdot \frac{1-\left(\mathbf{q}^{2} \mathbf{t}\right)^{n-1}}{1-\mathbf{q}^{4} \mathbf{t}^{2}}\right)
\end{aligned}
$$


what coincides with (4.7). For the dual branch we have

$$
\begin{aligned}
\tilde{\alpha} \cdot \lambda^{n}+\left.\tilde{\beta} \cdot \lambda^{3 n}\right|_{n= \pm 1}=\tilde{\mathcal{K}}_{u r}^{\text {unknot }}=(-\mathbf{t})^{\frac{n+1}{2}} \cdot \mathbf{q}+\left.\mathbf{q}^{-1}\right|_{n= \pm 1} & \Longrightarrow \\
\Longrightarrow \quad u_{u r} \tilde{\mathcal{K}}_{\square}^{[2, n]} & =\left(-\mathbf{q}^{2} \mathbf{t}\right)^{\frac{n-1}{2}} \cdot\left(-\mathbf{q} \mathbf{t}+\mathbf{q}^{-1}+\mathbf{q}^{3} \mathbf{t}^{2}\left(1+\mathbf{q}^{4} \mathbf{t}\right) \cdot \frac{1-\left(\mathbf{q}^{2} \mathbf{t}\right)^{n-1}}{1-\left(\mathbf{q}^{2} \mathbf{t}\right)^{2}}\right)= \\
& =\left(\left(-\mathbf{q}^{2} \mathbf{t}\right)\right)^{\frac{n+1}{2}} \cdot\left(\mathbf{q}+\mathbf{q}^{-1}-\frac{1+\mathbf{q}^{4} \mathbf{t}}{\mathbf{q}^{\mathbf{3}} \mathbf{t}} \cdot \frac{1-\left(\mathbf{q}^{2} \mathbf{t}\right)^{n+1}}{1-\mathbf{q}^{4} \mathbf{t}^{2}}\right)
\end{aligned}
$$

$\mathcal{K}_{u r}^{[2, n]}$ and $\tilde{\mathcal{K}}_{u r}^{[2, n]}$ are fully positive (or fully negative) respectively for $n>0$ and $n<0$, while in the "foreign" domains they contain terms with different signs — still they remain related by (4.13).

There is also a direct way from (4.15) to (4.16) and (4.17): instead of just multiplying by $[2]=\mathbf{q}+\mathbf{q}^{-1}$ one should apply the rule (4.4):

$$
[2] \cdot\left(1+\mathbf{q}^{2} \mathbf{t}\right) \longrightarrow \frac{1}{\mathbf{q}} \cdot\left(1+\mathbf{q}^{4} \mathbf{t}\right)
$$

Then we get from the first version of (4.15)

$$
[2] \cdot\left(1+\mathbf{q}^{4} \mathbf{t}^{2}\left(1+\mathbf{q}^{2} \mathbf{t}\right) \frac{1-\left(\mathbf{q}^{2} \mathbf{t}\right)^{n-1}}{1-\left(\mathbf{q}^{2} \mathbf{t}\right)^{2}}\right) \rightarrow \mathbf{q}+\mathbf{q}^{-1}+\mathbf{q}^{3} \mathbf{t}^{2}\left(1+\mathbf{q}^{4} \mathbf{t}\right) \frac{1-\left(\mathbf{q}^{2} \mathbf{t}\right)^{n-1}}{1-\left(\mathbf{q}^{2} \mathbf{t}\right)^{2}}
$$

i.e., exactly (4.16), while the second version turns into (4.17).

\section{Recursion for Khovanov $(N=2)$ polynomials at $m=3$}

In this case recursion is almost as simple as at $m=2$. For $N=2$ the r.h.s. with $\left\{A / t^{2}\right\} \stackrel{A=t^{2}}{=} 0$ in (3.5) can be omitted, but at the l.h.s. one should substitute $\mathbf{a}=\mathbf{q}^{2}$, i.e., $(A / t)^{6}=-\mathbf{q}^{6} \mathbf{t}^{3}$ : this is a manifestation of the double-face reduction rule (3.8). Thus, though this does not yet follow from any first principle argument, one can expect that Khovanov polynomials at $N=2$ satisfy the second order homogeneous equation

$$
\hat{\nabla}_{-\mathbf{q}^{6} \mathbf{t}^{3}}^{(3)} \hat{\nabla}_{-\mathbf{q}^{12} \mathbf{t}^{\mathbf{7}}}^{(3)} \mathcal{K}_{\square}^{[3, n]}(N=2)=0
$$

while Khovanov-Rozansky at $N=3$ should satisfy the full-fledged third order one, the same as the full superpolynomials with $\mathbf{a}=\mathbf{q}^{3}$ and $(A / t)^{6}=-\mathbf{q}^{\mathbf{1 2}} \mathbf{t}^{\mathbf{3}}$ :

$$
\hat{\nabla}_{-\mathbf{q}^{12} \mathbf{t}^{3}}^{(3)} \hat{\nabla}_{-\mathbf{q}^{\mathbf{1 8}} \mathbf{t}^{\mathbf{7}}}^{(3)} \hat{\nabla}_{-\mathbf{q}^{\mathbf{2 4}} \mathbf{t}^{\mathbf{9}}}^{(3)} \mathcal{K}_{\square}^{[3, n]}(N=3)=0
$$

We return to KR at $N=3$ in section 9 below, and concentrate in this section on Khovanov polynomials with $N=2$.

Making use of explicit expressions [32, 71] we can check that this short-evolution hypothesis is indeed true and restore entire evolution formulas:

$$
{ }_{r} \mathcal{K}_{\square}^{[3, n]}=\left\{\begin{array}{cc}
\left(-\mathbf{q}^{2} \mathbf{t}\right)^{n-1} \cdot\left(1+\left(1+\mathbf{q}^{2} \mathbf{t}+\mathbf{q}^{2} \mathbf{t}^{2}+\mathbf{q}^{6} \mathbf{t}^{3}\right) \cdot \mathbf{q}^{4} \mathbf{t}^{2} \cdot \frac{1-\left(\mathbf{q}^{6} \mathbf{t}^{4}\right)^{\frac{n-1}{3}}}{1-\mathbf{q}^{6} \mathbf{t}^{4}}\right) & n=1 \bmod 3 \\
\left(-\mathbf{q}^{2} \mathbf{t}\right)^{n+1} \cdot(-\mathbf{t})^{-1} \cdot\left(1-\frac{1+\mathbf{q}^{4} \mathbf{t}+\mathbf{q}^{4} \mathbf{t}^{2}+\mathbf{q}^{6} \mathbf{t}^{3}}{\mathbf{q}^{4} \mathbf{t}} \cdot \frac{1-\left(\mathbf{q}^{6} \mathbf{t}^{4}\right)^{\frac{n+1}{3}}}{1-\mathbf{q}^{6} \mathbf{t}^{4}}\right) & n=-1 \bmod 3
\end{array}\right.
$$


With the help of (4.15) we can check the topological invariance under the change $[m, n] \longleftrightarrow$ $[n, m]$ :

$$
\mathcal{K}_{r}^{[3,2]}=\mathcal{K}_{r}^{[2,3]}
$$

However, there is a non-trivial factor in the mirror-symmetry relation:

$$
\mathcal{K}_{r}^{[3,-n]}(\mathbf{q}, \mathbf{t})=-\frac{1}{\mathbf{t}} \cdot \mathcal{K}_{r}^{[3, n]}\left(\frac{1}{\mathbf{q}}, \frac{1}{\mathbf{t}}\right)
$$

and, accordingly, $\mathcal{K}_{r}^{[3,-2]}=-\frac{1}{\mathbf{t}} \cdot \mathcal{K}_{r}^{[3,2]}=-\frac{1}{\mathbf{t}} \cdot \mathcal{K}_{r}^{[2,3]}=-\frac{1}{\mathbf{t}} \cdot \mathcal{K}_{r}^{[2,-3]}$.

Similarly, for unreduced Khovanov polynomials we get:

$$
{ }_{u r} \mathcal{K}_{\square}^{[3, n]}=\left\{\begin{array}{cc}
\left(-\mathbf{q}^{2} \mathbf{t}\right)^{n-1} \cdot\left(\mathbf{q}+\mathbf{q}^{-1}+\left(1+\mathbf{q}^{2} \mathbf{t}^{2}+\mathbf{q}^{4} \mathbf{t}^{2}\right)\left(1+\mathbf{q}^{4} \mathbf{t}\right) \cdot \mathbf{q}^{3} \mathbf{t}^{2} \cdot \frac{1-\left(\mathbf{q}^{6} \mathbf{t}^{4}\right)^{\frac{n-1}{3}}}{1-\mathbf{q}^{6} \mathbf{t}^{4}}\right) & n=1 \bmod 3 \\
\left(-\mathbf{q}^{2} \mathbf{t}\right)^{n+1} \cdot(-\mathbf{t})^{-1} \cdot\left(\mathbf{q}+\mathbf{q}^{-1}-\frac{\left(1+\mathbf{q}^{2}+\mathbf{q}^{4} \mathbf{t}^{2}\right)\left(1+\mathbf{q}^{4} \mathbf{t}\right)}{\mathbf{q}^{5} \mathbf{t}} \cdot \frac{1-\left(\mathbf{q}^{6} \mathbf{t}^{4}\right)^{\frac{n+1}{3}}}{1-\mathbf{q}^{6} \mathbf{t}^{4}}\right) & n=-1 \bmod 3
\end{array}\right.
$$

Again, these formulas can be obtained directly from (5.3) by application of the rule (4.18), e.g.,

$$
\begin{aligned}
& {[2] \cdot\left(1+\left(\left(1+\mathbf{q}^{2} \mathbf{t}\right)+\mathbf{q}^{2} \mathbf{t}^{2}\left(1+\mathbf{q}^{4} \mathbf{t}\right)\right) \cdot \mathbf{q}^{4} \mathbf{t}^{2} \cdot \frac{1-\left(\mathbf{q}^{6} \mathbf{t}^{4}\right)^{\frac{n-1}{3}}}{1-\mathbf{q}^{6} \mathbf{t}^{4}}\right)} \\
& \longrightarrow \mathbf{q}+\mathbf{q}^{-1}+\left(1+\mathbf{q}^{4} \mathbf{t}\right)\left(1+[2] \mathbf{q}^{3} \mathbf{t}^{2}\right) \cdot \mathbf{q}^{4} \mathbf{t}^{2} \cdot \frac{1-\left(\mathbf{q}^{6} \mathbf{t}^{4}\right)^{\frac{n-1}{3}}}{1-\mathbf{q}^{6} \mathbf{t}^{4}}
\end{aligned}
$$

Unreduced polynomials satisfy topological identity

$$
\mathcal{K}_{u r}^{[3,2]}=\mathcal{K}_{u r}^{[2,3]}
$$

and mirror relation

$$
\mathcal{K}_{u r}^{[3,-n]}(\mathbf{q}, \mathbf{t})=-\frac{1}{\mathbf{t}} \cdot \mathcal{K}_{u r}^{[3, n]}\left(\frac{1}{\mathbf{q}}, \frac{1}{\mathbf{t}}\right)
$$

\section{Recursion for Khovanov $(N=2)$ polynomials at $m=4$}

Proceeding to four strands, $m=4$, we can expect the third-order difference equation for Khovanov polynomials,

$$
\hat{\nabla}_{\mathbf{q}^{12} \mathbf{t}^{6}}^{(4)} \hat{\nabla}_{\mathbf{q}^{20} \mathbf{t}^{12}}^{(4)} \hat{\nabla}_{\mathbf{q}^{24} \mathbf{t}^{14}}^{(4)} \mathcal{K}_{\square}^{[4, n]}(N=2)=0
$$

This means that they should be linear combinations of three Lyapunov exponentials and indeed they are. 
For $n=1 \bmod 4$

$$
\begin{aligned}
& \mathcal{K}_{r}^{[4, n]}=\left(-\mathbf{q}^{\mathbf{2}} \mathbf{t}\right)^{\frac{3(n-1)}{2}} \cdot\left(1-\frac{(1+\mathbf{t}) \cdot \mathbf{q}^{\mathbf{1 0}} \mathbf{t}^{\mathbf{5}}\left(1+\mathbf{q}^{\mathbf{2}} \mathbf{t}\right) \cdot \frac{1-\left(\mathbf{q}^{\mathbf{1 2}} \mathbf{t}^{\mathbf{8}}\right)^{\frac{n-1}{4}}}{\left(1-\mathbf{q}^{\mathbf{4}} \mathbf{t}^{\mathbf{2}}\right)\left(1-\mathbf{q}^{\mathbf{6}} \mathbf{t}^{\mathbf{4}}\right)}}{(1-}\right. \\
& \left.+\mathbf{q}^{4} \mathbf{t}^{2}\left(1+\mathbf{q}^{\mathbf{6}} \mathbf{t}^{\mathbf{3}}\right)\left(1+\frac{\mathbf{q}^{2} \mathbf{t}\left(1+\mathbf{t}+\mathbf{q}^{2} \mathbf{t}^{3}+\mathbf{q}^{6} \mathbf{t}^{4}\right)}{1-\mathbf{q}^{4} \mathbf{t}^{2}}\right) \cdot \frac{1-\left(\mathbf{q}^{8} \mathbf{t}^{\mathbf{6}}\right)^{\frac{n-1}{4}}}{1-\mathbf{q}^{8} \mathbf{t}^{\mathbf{6}}}\right) \\
& \mathcal{K}_{u r}^{[4, n]}=\left(-\mathbf{q}^{2} \mathbf{t}\right)^{\frac{3(n-1)}{2}} \cdot\left(\mathbf{q}+\mathbf{q}^{-1}-\underline{(1+\mathbf{t}) \cdot \mathbf{q}^{\mathbf{9}} \mathbf{t}^{\mathbf{5}}\left(1+\mathbf{q}^{\mathbf{4}} \mathbf{t}\right) \cdot \frac{1-\left(\mathbf{q}^{\mathbf{1 2}} \mathbf{t}^{\mathbf{8}}\right)^{\frac{n-1}{4}}}{\left(1-\mathbf{q}^{\mathbf{4}} \mathbf{t}^{\mathbf{2}}\right)\left(1-\mathbf{q}^{\mathbf{6}} \mathbf{t}^{\mathbf{4}}\right)}}+\right. \\
& \left.+\left(\mathbf{q}^{3} \mathbf{t}^{2}\left(1+\mathbf{q}^{2} \mathbf{t}^{2}\right)\left(1+\mathbf{q}^{4} \mathbf{t}^{2}\right)+\frac{\mathbf{q}^{7} \mathbf{t}^{5}\left(\mathbf{t}+\mathbf{q}^{2}+\mathbf{q}^{2} \mathbf{t}+\mathbf{q}^{8} \mathbf{t}^{4}\right)}{1-\mathbf{q}^{4} \mathbf{t}^{2}}\right) \cdot\left(1+\mathbf{q}^{4} \mathbf{t}\right) \cdot \frac{1-\left(\mathbf{q}^{8} \mathbf{t}^{6}\right)^{\frac{n-1}{4}}}{1-\mathbf{q}^{8} \mathbf{t}^{6}}\right)
\end{aligned}
$$

Underlined structures enter with the coefficient $(1+\mathbf{t})$, because it does not contribute in the case of HOMFLY polynomial (the coefficient in front of $D_{[22]}$ is "accidentally" vanishing when $t=q$ ). Despite it inters with negative sign, it does not spoil the positivity of the entire expression at $n>0$. However, at $n<0$ the polynomial fail to be positive or negative and consists of monomials with different signs. Thus there are no chances for any relation like (1.6).

For $n=-1 \bmod 4$

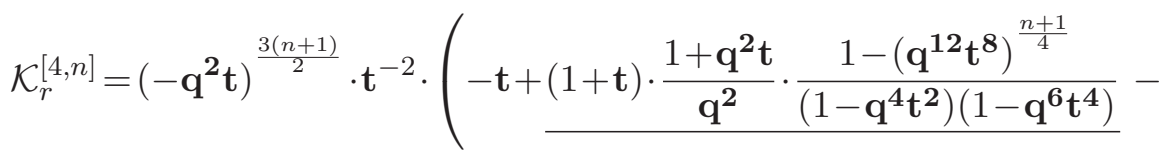

$$
\begin{aligned}
& \left.-\frac{1+\mathbf{q}^{6} \mathbf{t}^{3}}{\mathbf{q}^{6} \mathbf{t}} \cdot \frac{1+\mathbf{q}^{4} \mathbf{t}+\mathbf{q}^{4} \mathbf{t}^{2}\left(1-\mathbf{q}^{4} \mathbf{t}^{2}\right)+\mathbf{q}^{6} \mathbf{t}^{3}(1+\mathbf{t})}{1-\mathbf{q}^{4} \mathbf{t}^{2}} \cdot \frac{1-\left(\mathbf{q}^{8} \mathbf{t}^{6}\right)^{\frac{n+1}{4}}}{1-\mathbf{q}^{8} \mathbf{t}^{6}}\right) \\
& \mathcal{K}_{u r}^{[4, n]}=\left(-\mathbf{q}^{2} \mathbf{t}\right)^{\frac{3(n+1)}{2}} \cdot \mathbf{t}^{-2} \cdot\left(-\mathbf{q} \mathbf{t}+\mathbf{q}^{-1}+\underline{(1+\mathbf{t}) \cdot \frac{1+\mathbf{q}^{\mathbf{4}} \mathbf{t}}{\mathbf{q}^{\mathbf{3}}} \cdot \frac{1-\left(\mathbf{q}^{12} \mathbf{t}^{\mathbf{8}}\right)^{\frac{n+1}{4}}}{\left(1-\mathbf{q}^{\mathbf{4}} \mathbf{t}^{\mathbf{2}}\right)\left(1-\mathbf{q}^{\mathbf{6}} \mathbf{t}^{\mathbf{4}}\right)}}-\right. \\
& \left.-\left(1+\mathbf{q}^{4} \mathbf{t}\right) \cdot \frac{1+\mathbf{q}^{2}\left(1-\mathbf{q}^{4} \mathbf{t}^{2}\right)+\mathbf{q}^{4} \mathbf{t}^{2}+\mathbf{q}^{6} \mathbf{t}^{4}+\mathbf{q}^{8} \mathbf{t}^{3}+\mathbf{q}^{10} \mathbf{t}^{5}-\mathbf{q}^{12} \mathbf{t}^{6}}{\mathbf{q}^{7} \mathbf{t}\left(1-\mathbf{q}^{4} \mathbf{t}^{2}\right)} \cdot \frac{1-\left(\mathbf{q}^{8} \mathbf{t}^{6}\right)^{\frac{n+1}{4}}}{1-\mathbf{q}^{8} \mathbf{t}^{6}}\right)
\end{aligned}
$$

Despite this is not evident from the formulas, for $n>0$ (but not for $n<0$ ) these polynomials are pure positive or pure negative, depending on parity of $\frac{n+1}{2}$. As already mentioned, this time there is no relation like (5.5):

$$
\mathcal{K}_{\square}^{[4,-n]}(\mathbf{q}, \mathbf{t}) \nsucc \mathcal{K}_{\square}^{[4, n]}\left(\frac{1}{\mathbf{q}}, \frac{1}{\mathbf{t}}\right)
$$

neither in unreduced nor in reduced case, moreover, the 1.h.s. is not even a positive Laurent polynomial. The valid topological identity is

$$
\mathcal{K}_{\square}^{[4,3]}=\mathcal{K}_{\square}^{[3,4]}
$$


Also, it is unclear how the rule (4.4) can be applied to derive unreduced polynomials from the reduced ones. This drawback is cured by an improved (better structured) version of above formulas - which one day should be directly deduced from an adequately structured expression for the superpolynomials.

For $n=1 \bmod 4$ :

$$
\begin{aligned}
& \mathcal{K}_{r}^{[4, n]}=\left(-\mathbf{q}^{2} \mathbf{t}\right)^{\frac{3(n-1)}{2}} \\
& \left(1+\left(1+\mathbf{q}^{2} \mathbf{t}\right) \cdot \mathbf{q}^{4} \mathbf{t}^{2}\left(1+\mathbf{q}^{8} \mathbf{t}^{\mathbf{6}}\right) \cdot \frac{1-\left(\mathbf{q}^{8} \mathbf{t}^{6}\right)^{\frac{n-1}{4}}}{1-\mathbf{q}^{\mathbf{8}} \mathbf{t}^{\mathbf{2}}}+\left(1+\mathbf{q}^{4} \mathbf{t}\right) \cdot \mathbf{q}^{6} \mathbf{t}^{4}\left(1+\mathbf{q}^{2} \mathbf{t}^{2}\right) \cdot \frac{1-\left(\mathbf{q}^{8} \mathbf{t}^{6}\right)^{\frac{n-1}{4}}}{1-\mathbf{q}^{8} \mathbf{t}^{6}}+\right. \\
& \left.+\left(1+\mathbf{q}^{2} \mathbf{t}\right) \cdot \frac{\mathbf{q}^{18} \mathbf{t}^{11}(1+\mathbf{t})}{1-\mathbf{q}^{\mathbf{6}} \mathbf{t}^{4}} \cdot \frac{1-\left(\mathbf{q}^{8} \mathbf{t}^{\mathbf{6}}\right)^{\frac{n-1}{4}}}{1-\mathbf{q}^{8} \mathbf{t}^{6}}-\left(1+\mathbf{q}^{2} \mathbf{t}\right) \cdot \frac{\mathbf{q}^{10} \mathbf{t}^{\mathbf{5}}(1+\mathbf{t})}{1-\mathbf{q}^{6} \mathbf{t}^{4}} \cdot \frac{1-\left(\mathbf{q}^{4} \mathbf{t}^{2}\right)^{\frac{n-1}{4}}}{1-\mathbf{q}^{4} \mathbf{t}^{2}} \cdot\left(\mathbf{q}^{8} \mathbf{t}^{\mathbf{6}}\right)^{\frac{n-1}{4}}\right)
\end{aligned}
$$

Together the two items in the second line form a positive polynomial, but this positivity is still not explicit. But now the unreduced polynomial is obtained by direct application of (4.18):

$$
\begin{aligned}
& \mathcal{K}_{u r}^{[4, n]}=\left(-\mathbf{q}^{2} \mathbf{t}\right)^{\frac{3(n-1)}{2}} \\
& \cdot\left([2]+\left(1+\mathbf{q}^{4} \mathbf{t}\right) \cdot \mathbf{q}^{3} \mathbf{t}^{2}\left(1+\mathbf{q}^{8} \mathbf{t}^{6} \cdot \frac{1-\left(\mathbf{q}^{8} \mathbf{t}^{6}\right)^{\frac{n-1}{4}}}{1-\mathbf{q}^{8} \mathbf{t}^{6}}+[2] \cdot\left(1+\mathbf{q}^{4} \mathbf{t}\right) \cdot \mathbf{q}^{6} \mathbf{t}^{4}\left(1+\mathbf{q}^{2} \mathbf{t}^{2}\right) \cdot \frac{1-\left(\mathbf{q}^{8} \mathbf{t}^{6}\right)^{\frac{n-1}{4}}}{1-\mathbf{q}^{8} \mathbf{t}^{6}}+\right.\right. \\
& \left.+\left(1+\mathbf{q}^{4} \mathbf{t}\right) \cdot \frac{\mathbf{q}^{17} \mathbf{t}^{11}(1+\mathbf{t})}{1-\mathbf{q}^{6} \mathbf{t}^{4}} \cdot \frac{1-\left(\mathbf{q}^{8} \mathbf{t}^{6}\right)^{\frac{n-1}{4}}}{1-\mathbf{q}^{8} \mathbf{t}^{6}}-\left(1+\mathbf{q}^{4} \mathbf{t}\right) \cdot \frac{\mathbf{q}^{9} \mathbf{t}^{5}(1+\mathbf{t})}{1-\mathbf{q}^{6} \mathbf{t}^{4}} \cdot \frac{1-\left(\mathbf{q}^{4} \mathbf{t}^{2}\right)^{\frac{n-1}{4}}}{1-\mathbf{q}^{4} \mathbf{t}^{2}} \cdot\left(\mathbf{q}^{8} \mathbf{t}^{6}\right)^{\frac{n-1}{4}}\right)
\end{aligned}
$$

The analogues of these formulas for $n=-1 \bmod 4$ are a little more complicated:

$$
\begin{aligned}
& \mathcal{K}_{r}^{[4, n]}=\left(-\mathbf{q}^{2} \mathbf{t}\right)^{\frac{3(n-1)}{2}} \cdot\left(1+\left(\left(1+\mathbf{q}^{2} \mathbf{t}\right) \cdot \mathbf{q}^{4} \mathbf{t}^{2}+\left(1+\mathbf{q}^{4} \mathbf{t}\right) \cdot \mathbf{q}^{6} \mathbf{t}^{4}\right) \cdot \frac{1-\left(\mathbf{q}^{8} \mathbf{t}^{6}\right)^{\frac{n+1}{4}}}{1-\mathbf{q}^{8} \mathbf{t}^{6}}+\right. \\
& +\left(\left(1+\mathbf{q}^{2} \mathbf{t}\right) \cdot \mathbf{q}^{12} \mathbf{t}^{8}+\left(1+\mathbf{q}^{4} \mathbf{t}\right) \cdot \mathbf{q}^{8} \mathbf{t}^{6}\right) \cdot \frac{1-\left(\mathbf{q}^{8} \mathbf{t}^{6}\right)^{\frac{n-3}{4}}}{1-\mathbf{q}^{8} \mathbf{t}^{6}}+ \\
& \left.+\left(1+\mathbf{q}^{2} \mathbf{t}\right) \cdot \frac{\mathbf{q}^{18} \mathbf{t}^{11}(1+\mathbf{t})}{1-\mathbf{q}^{6} \mathbf{t}^{4}} \cdot \frac{1-\left(\mathbf{q}^{8} \mathbf{t}^{6}\right)^{\frac{n-3}{4}}}{1-\mathbf{q}^{8} \mathbf{t}^{6}}-\left(1+\mathbf{q}^{2} \mathbf{t}\right) \cdot \frac{\mathbf{q}^{8} \mathbf{t}^{3}(1+\mathbf{t})}{1-\mathbf{q}^{6} \mathbf{t}^{4}} \cdot \frac{1-\left(\mathbf{q}^{4} \mathbf{t}^{2}\right)^{\frac{n-3}{4}}}{1-\mathbf{q}^{4} \mathbf{t}^{2}} \cdot\left(\mathbf{q}^{8} \mathbf{t}^{\mathbf{6}}\right)^{\frac{n+1}{4}}\right)
\end{aligned}
$$

and

$$
\begin{aligned}
& \mathcal{K}_{u r}^{[4, n]}=\left(-\mathbf{q}^{2} \mathbf{t}\right)^{\frac{3(n-1)}{2}} \cdot\left([2]+\left(1+\mathbf{q}^{4} \mathbf{t}\right) \cdot\left(\mathbf{q}^{3} \mathbf{t}^{2}+[2] \cdot \mathbf{q}^{6} \mathbf{t}^{4}\right) \cdot \frac{1-\left(\mathbf{q}^{8} \mathbf{t}^{6}\right)^{\frac{n+1}{4}}}{1-\mathbf{q}^{8} \mathbf{t}^{6}}\right. \\
& +\left(1+\mathbf{q}^{4} \mathbf{t}\right) \cdot\left(\mathbf{q}^{11} \mathbf{t}^{8}+[2] \cdot \mathbf{q}^{8} \mathbf{t}^{\mathbf{6}}\right) \cdot \frac{1-\left(\mathbf{q}^{8} \mathbf{t}^{6}\right)^{\frac{n-3}{4}}}{1-\mathbf{q}^{8} \mathbf{t}^{6}}+ \\
& \left.+\left(1+\mathbf{q}^{4} \mathbf{t}\right) \cdot \frac{\mathbf{q}^{17} \mathbf{t}^{11}(1+\mathbf{t})}{1-\mathbf{q}^{6} \mathbf{t}^{4}} \cdot \frac{1-\left(\mathbf{q}^{8} \mathbf{t}^{\mathbf{6}}\right)^{\frac{n-3}{4}}}{1-\mathbf{q}^{8} \mathbf{t}^{\mathbf{6}}}-\left(1+\mathbf{q}^{4} \mathbf{t}\right) \cdot \frac{\mathbf{q}^{7} \mathbf{t}^{3}(1+\mathbf{t})}{1-\mathbf{q}^{6} \mathbf{t}^{4}} \cdot \frac{1-\left(\mathbf{q}^{4} \mathbf{t}^{2}\right)^{\frac{n-3}{4}}}{1-\mathbf{q}^{4} \mathbf{t}^{2}} \cdot\left(\mathbf{q}^{8} \mathbf{t}^{\mathbf{6}}\right)^{\frac{n+1}{4}}\right)
\end{aligned}
$$

\section{The case of $m=5$}

This time there are four different kinds of formulas, for four different residues $n=$ 1,2,3,4 $\bmod 5$. The corresponding formulas for Khovanov polynomials are: 


\section{Residue 1.}

$$
\begin{aligned}
& \mathcal{K}_{r}^{[5, n]}=\left(-\mathbf{q}^{2} \mathbf{t}\right)^{2(n-1)} \\
& \cdot\left(1-\frac{\mathbf{q}^{10} \mathbf{t}^{5}\left(1+\mathbf{q}^{8} \mathbf{t}^{\mathbf{6}}\right)\left(\left(1+\mathbf{q}^{2} \mathbf{t}\right)\left(1+\mathbf{t}+\mathbf{q}^{2} \mathbf{t}^{3}+\mathbf{q}^{\mathbf{6}} \mathbf{t}^{\mathbf{4}}\right)+\left(1+\mathbf{q}^{4} \mathbf{t}\right) \mathbf{q}^{2} \mathbf{t}^{2}\left(1+\mathbf{q}^{2} \mathbf{t}^{3}\right)\right)}{1-\mathbf{q}^{\mathbf{6}} \mathbf{t}^{4}} \cdot \frac{1-\left(\mathbf{q}^{\mathbf{1 6}} \mathbf{t}^{\mathbf{1 2}}\right)^{\frac{n-1}{4}}}{1-\mathbf{q}^{\mathbf{1 6}} \mathbf{t}^{\mathbf{1 2}}}+\right. \\
& +\left(+\frac{\left(1+\mathbf{q}^{2} \mathbf{t}\right) \cdot \mathbf{q}^{4} \mathbf{t}^{2}\left(1+\mathbf{q}^{6} \mathbf{t}^{3}+2 \mathbf{q}^{8} \mathbf{t}^{6}+\mathbf{q}^{10} \mathbf{t}^{8}+\mathbf{q}^{12} \mathbf{t}^{7}+2 \mathbf{q}^{14} \mathbf{t}^{9}\right)}{1-\mathbf{q}^{6} \mathbf{t}^{4}}\right. \\
& \left.\left.+\frac{\left(1+\mathbf{q}^{4} \mathbf{t}\right) \cdot \mathbf{q}^{6} \mathbf{t}^{4}\left(1+\mathbf{q}^{2} \mathbf{t}^{2}+\mathbf{q}^{4} \mathbf{t}^{4}+\mathbf{q}^{6} \mathbf{t}^{3}-\mathbf{q}^{6} \mathbf{t}^{4}+\mathbf{q}^{14} \mathbf{t}^{9}\right)}{1-\mathbf{q}^{6} \mathbf{t}^{4}}\right) \cdot \frac{1-\left(\mathbf{q}^{10} \mathbf{t}^{8}\right)^{\frac{n-1}{4}}}{1-\mathbf{q}^{10} \mathbf{t}^{8}}\right) \\
& \mathcal{K}_{u r}^{[5, n]}=\left(-\mathbf{q}^{2} \mathbf{t}\right)^{2(n-1)}
\end{aligned}
$$

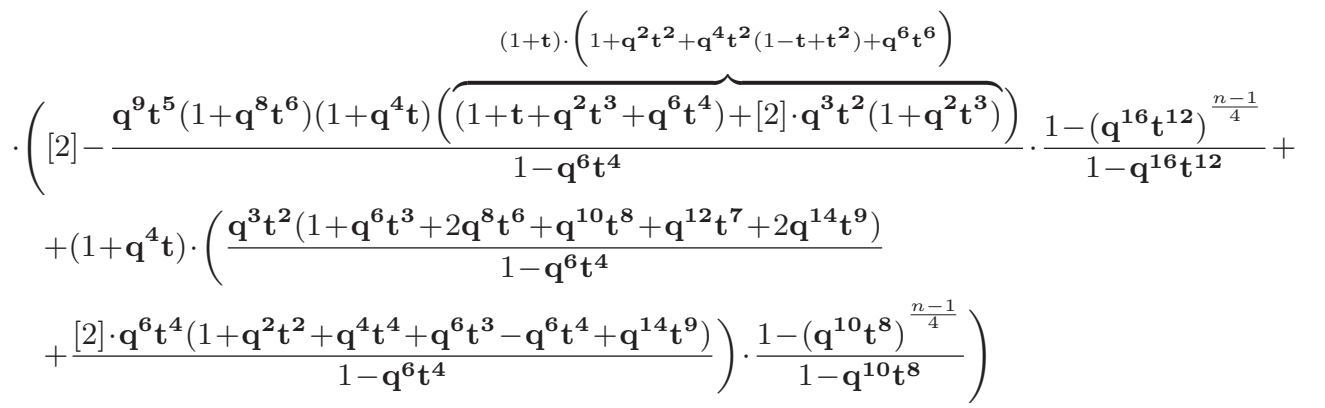

Residue 2.

$$
\begin{aligned}
& \mathcal{K}_{r}^{[5, n]}=\left(-\mathbf{q}^{2} \mathbf{t}\right)^{2(n-1)} \cdot\left(1+\mathbf{q}^{4} \mathbf{t}^{2}\left(1+\mathbf{q}^{2} \mathbf{t}\right)\left(1+\mathbf{q}^{4} \mathbf{t}^{2}\right)-\right. \\
& -\frac{\mathbf{q}^{12} \mathbf{t}^{7}\left(1+\mathbf{q}^{8} \mathbf{t}^{6}\right)\left(\left(1+\mathbf{q}^{2} \mathbf{t}\right)\left[(1+\mathbf{t})\left(\mathbf{q}^{10} \mathbf{t}^{6}+\mathbf{q}^{6} \mathbf{t}^{4}-\mathbf{q}^{2}\right)+\mathbf{t}\left(1+\mathbf{q}^{4} \mathbf{t}\right)\right]+\left(1+\mathbf{q}^{4} \mathbf{t}\right)\left(1+\mathbf{q}^{2} \mathbf{t}^{3}\right)\right)}{1-\mathbf{q}^{6} \mathbf{t}^{4}} \\
& \frac{1-\left(\mathbf{q}^{\mathbf{1 6}} \mathbf{t}^{\mathbf{1 2}}\right)^{\frac{n-2}{4}}}{1-\mathbf{q}^{\mathbf{1 6}} \mathbf{t}^{\mathbf{1 2}}} \\
& +\left[\frac{\left(1+\mathbf{q}^{2} \mathbf{t}\right) \cdot \mathbf{q}^{8} \mathbf{t}^{4}\left(-1+2 \mathbf{q}^{4} \mathbf{t}^{4}-\mathbf{q}^{6} \mathbf{t}^{3}+2 \mathbf{q}^{6} \mathbf{t}^{6}+\mathbf{q}^{8} \mathbf{t}^{5}+2 \mathbf{q}^{10} \mathbf{t}^{7}+\mathbf{q}^{10} \mathbf{t}^{8}+\mathbf{q}^{12} \mathbf{t}^{9}+\mathbf{q}^{16} \mathbf{t}^{11}\right)}{1-\mathbf{q}^{6} \mathbf{t}^{4}}+\right. \\
& \left.\left.+\frac{\left(1+\mathbf{q}^{4} \mathbf{t}\right) \cdot \mathbf{q}^{6} \mathbf{t}^{4}\left(1+\mathbf{q}^{2} \mathbf{t}^{2}+\mathbf{q}^{4} \mathbf{t}^{4}+\mathbf{q}^{6} \mathbf{t}^{3}-\mathbf{q}^{6} \mathbf{t}^{4}+\mathbf{q}^{14} \mathbf{t}^{9}\right)}{1-\mathbf{q}^{6} \mathbf{t}^{4}}\right] \cdot \frac{1-\left(\mathbf{q}^{10} \mathbf{t}^{8}\right)^{\frac{n-2}{4}}}{1-\mathbf{q}^{10} \mathbf{t}^{8}}\right) \\
& \mathcal{K}_{u r}^{[5, n]}=\left(-\mathbf{q}^{2} \mathbf{t}\right)^{2(n-1)} \cdot\left([2]+\mathbf{q}^{3} \mathbf{t}^{2}\left(1+\mathbf{q}^{4} \mathbf{t}\right)\left(1+\mathbf{q}^{4} \mathbf{t}^{2}\right)-\right. \\
& -\frac{\mathbf{q}^{11} \mathbf{t}^{\mathbf{7}}\left(1+\mathbf{q}^{8} \mathbf{t}^{\mathbf{6}}\right)\left(1+\mathbf{q}^{4} \mathbf{t}\right)\left(\left[(1+\mathbf{t})\left(\mathbf{q}^{10} \mathbf{t}^{\mathbf{6}}+\mathbf{q}^{\mathbf{6}} \mathbf{t}^{4}-\mathbf{q}^{2}\right)+\mathbf{t}\left(1+\mathbf{q}^{4} \mathbf{t}\right)\right]+[2] \cdot \mathbf{q}\left(1+\mathbf{q}^{2} \mathbf{t}^{3}\right)\right)}{1-\mathbf{q}^{\mathbf{6}} \mathbf{t}^{4}} \cdot \frac{1-\left(\mathbf{q}^{\mathbf{1 6}} \mathbf{t}^{12}\right)^{\frac{n-2}{4}}}{1-\mathbf{q}^{\mathbf{1 6}} \mathbf{t}^{12}}+ \\
& +\left(1+\mathbf{q}^{4} \mathbf{t}\right) \cdot\left[\frac{\mathbf{q}^{7} \mathbf{t}^{4}\left(-1+2 \mathbf{q}^{4} \mathbf{t}^{4}-\mathbf{q}^{6} \mathbf{t}^{3}+2 \mathbf{q}^{6} \mathbf{t}^{6}+\mathbf{q}^{8} \mathbf{t}^{5}+2 \mathbf{q}^{10} \mathbf{t}^{7}+\mathbf{q}^{10} \mathbf{t}^{8}+\mathbf{q}^{12} \mathbf{t}^{9}+\mathbf{q}^{16} \mathbf{t}^{11}\right)}{1-\mathbf{q}^{6} \mathbf{t}^{4}}+\right. \\
& \left.\left.+\frac{[2] \cdot \mathbf{q}^{6} \mathbf{t}^{4}\left(1+\mathbf{q}^{2} \mathbf{t}^{2}+\mathbf{q}^{4} \mathbf{t}^{4}+\mathbf{q}^{6} \mathbf{t}^{3}-\mathbf{q}^{6} \mathbf{t}^{4}+\mathbf{q}^{14} \mathbf{t}^{9}\right)}{1-\mathbf{q}^{6} \mathbf{t}^{4}}\right] \cdot \frac{1-\left(\mathbf{q}^{10} \mathbf{t}^{8}\right)^{\frac{n-2}{4}}}{1-\mathbf{q}^{10} \mathbf{t}^{8}}\right)
\end{aligned}
$$




\section{Residue 3.}

$$
\begin{aligned}
& \mathcal{K}_{r}^{[5, n]}=\left(-\mathbf{q}^{2} \mathbf{t}\right)^{2(n-1)} \cdot\left(1+\left(1+\mathbf{q}^{2} \mathbf{t}\right) \cdot \mathbf{q}^{4} \mathbf{t}^{2}\left(1+\mathbf{q}^{6} \mathbf{t}^{4}\right)+\left(1+\mathbf{q}^{4} \mathbf{t}\right) \cdot \mathbf{q}^{6} \mathbf{t}^{4}-\right. \\
& -\frac{\left(\left(1+\mathbf{q}^{2} \mathbf{t}\right) \cdot\left(1-\mathbf{q}^{4} \mathbf{t}^{2}+\mathbf{q}^{6} \mathbf{t}^{3}+\mathbf{q}^{6} \mathbf{t}^{4}+\mathbf{q}^{12} \mathbf{t}^{7}+\mathbf{q}^{12} \mathbf{t}^{8}\right)+\left(1+\mathbf{q}^{4} \mathbf{t}\right) \cdot\left(-1+\mathbf{q}^{2} \mathbf{t}^{2}+\mathbf{q}^{8} \mathbf{t}^{5}+\mathbf{q}^{8} \mathbf{t}^{6}\right)\right)}{1-\mathbf{q}^{6} \mathbf{t}^{4}} \\
& \frac{\mathbf{q}^{12} \mathbf{t}^{8}\left(1+\mathbf{q}^{\mathbf{8}} \mathbf{t}^{\mathbf{6}}\right)}{1-\mathbf{q}^{\mathbf{6}} \mathbf{t}^{4}} \cdot \frac{1-\left(\mathbf{q}^{16} \mathbf{t}^{12}\right)^{\frac{n-3}{4}}}{1-\mathbf{q}^{16} \mathbf{t}^{12}}+ \\
& +\left[\frac{\left(1+\mathbf{q}^{2} \mathbf{t}\right) \cdot \mathbf{q}^{10} \mathbf{t}^{6}\left(-1+2 \mathbf{q}^{2} \mathbf{t}^{2}+2 \mathbf{q}^{4} \mathbf{t}^{4}+2 \mathbf{q}^{8} \mathbf{t}^{5}+\mathbf{q}^{10} \mathbf{t}^{7}+\mathbf{q}^{10} \mathbf{t}^{8}+\mathbf{q}^{16} \mathbf{t}^{11}\right)}{1-\mathbf{q}^{6} \mathbf{t}^{4}}+\right. \\
& \left.\left.+\frac{\left(1+\mathbf{q}^{4} \mathbf{t}\right) \cdot \mathbf{q}^{8} \mathbf{t}^{\mathbf{6}}\left(1+\mathbf{q}^{2} \mathbf{t}^{2}\right)\left(1+\mathbf{q}^{2} \mathbf{t}\right)\left(1-\mathbf{q}^{2} \mathbf{t}+\mathbf{q}^{6} \mathbf{t}^{4}-\mathbf{q}^{8} \mathbf{t}^{5}+\mathbf{q}^{10} \mathbf{t}^{6}\right)}{1-\mathbf{q}^{6} \mathbf{t}^{4}}\right] \cdot \frac{1-\left(\mathbf{q}^{10} \mathbf{t}^{8}\right)^{\frac{n-3}{4}}}{1-\mathbf{q}^{10} \mathbf{t}^{8}}\right) \\
& \mathcal{K}_{u r}^{[5, n]}=\left(-\mathbf{q}^{2} \mathbf{t}\right)^{2(n-1)} \cdot\left([2]+\left(1+\mathbf{q}^{4} \mathbf{t}\right) \cdot\left(\mathbf{q}^{3} \mathbf{t}^{2}\left(1+\mathbf{q}^{\mathbf{6}} \mathbf{t}^{\mathbf{4}}\right)+[2] \cdot \mathbf{q}^{6} \mathbf{t}^{4}\right)-\right. \\
& -\left(1+\mathbf{q}^{4} \mathbf{t}\right) \cdot \frac{\left(\left(1-\mathbf{q}^{4} \mathbf{t}^{2}+\mathbf{q}^{6} \mathbf{t}^{3}+\mathbf{q}^{6} \mathbf{t}^{4}+\mathbf{q}^{12} \mathbf{t}^{7}+\mathbf{q}^{12} \mathbf{t}^{8}\right)+[2] \mathbf{q} \cdot\left(-1+\mathbf{q}^{2} \mathbf{t}^{2}+\mathbf{q}^{8} \mathbf{t}^{5}+\mathbf{q}^{8} \mathbf{t}^{6}\right)\right) \cdot \mathbf{q}^{11} \mathbf{t}^{8}\left(1+\mathbf{q}^{8} \mathbf{t}^{6}\right)}{1-\mathbf{q}^{6} \mathbf{t}^{4}} \\
& \frac{1-\left(\mathbf{q}^{\mathbf{1 6}} \mathbf{t}^{12}\right)^{\frac{n-3}{4}}}{1-\mathbf{q}^{\mathbf{1 6}} \mathbf{t}^{\mathbf{1 2}}}+ \\
& +\left(1+\mathbf{q}^{4} \mathbf{t}\right) \cdot\left[\frac{\mathbf{q}^{9} \mathbf{t}^{6}\left(-1+2 \mathbf{q}^{2} \mathbf{t}^{2}+2 \mathbf{q}^{4} \mathbf{t}^{4}+2 \mathbf{q}^{8} \mathbf{t}^{5}+\mathbf{q}^{10} \mathbf{t}^{7}+\mathbf{q}^{10} \mathbf{t}^{8}+\mathbf{q}^{16} \mathbf{t}^{11}\right)}{1-\mathbf{q}^{6} \mathbf{t}^{4}}+\right. \\
& \left.\left.+[2] \cdot \frac{\mathbf{q}^{8} \mathbf{t}^{\mathbf{6}}\left(1+\mathbf{q}^{2} \mathbf{t}^{2}\right)\left(1+\mathbf{q}^{2} \mathbf{t}\right)\left(1-\mathbf{q}^{2} \mathbf{t}+\mathbf{q}^{6} \mathbf{t}^{4}-\mathbf{q}^{8} \mathbf{t}^{5}+\mathbf{q}^{10} \mathbf{t}^{6}\right)}{1-\mathbf{q}^{6} \mathbf{t}^{4}}\right] \cdot \frac{1-\left(\mathbf{q}^{10} \mathbf{t}^{8}\right)^{\frac{n-3}{4}}}{1-\mathbf{q}^{10} \mathbf{t}^{8}}\right)
\end{aligned}
$$

\section{Residue 4.}

$$
\begin{aligned}
& \mathcal{K}_{r}^{[5, n]}=\left(-\mathbf{q}^{2} \mathbf{t}\right)^{2(n-1)} \cdot\left(1+\left(1+\mathbf{q}^{2} \mathbf{t}\right) \cdot \mathbf{q}^{4} \mathbf{t}^{2}\left(1+\mathbf{q}^{8} \mathbf{t}^{\mathbf{6}}\right)+\left(1+\mathbf{q}^{4} \mathbf{t}\right) \cdot \mathbf{q}^{6} \mathbf{t}^{4}\left(1+\mathbf{q}^{2} \mathbf{t}^{2}\right)-\right. \\
& (1+\mathbf{t}) \cdot\left(\left(1+\mathbf{q}^{2} \mathbf{t}\right) \cdot \mathbf{q}^{6} \mathbf{t}^{4}+\left(1+\mathbf{q}^{4} \mathbf{t}\right) \cdot\left(1+\mathbf{q}^{2} \mathbf{t}^{2}\right)\right) \\
& -\frac{\mathbf{q}^{20} \mathbf{t}^{13}\left(1+\mathbf{q}^{8} \mathbf{t}^{6}\right) \cdot(\overbrace{\left(1+\mathbf{q}^{2} \mathbf{t}\right) \cdot \mathbf{t} \cdot\left(1+\mathbf{q}^{4} \mathbf{t}+\mathbf{q}^{6} \mathbf{t}^{3}+\mathbf{q}^{6} \mathbf{t}^{4}\right)+\left(1+\mathbf{q}^{4} \mathbf{t}\right) \cdot\left(1+\mathbf{q}^{2} \mathbf{t}^{3}\right)})}{1-\mathbf{q}^{6} \mathbf{t}^{4}} \cdot \frac{1-\left(\mathbf{q}^{16} \mathbf{t}^{12}\right)^{\frac{n-4}{4}}}{1-\mathbf{q}^{16} \mathbf{t}^{12}}+ \\
& +\left[\frac{\left(1+\mathbf{q}^{2} \mathbf{t}\right) \cdot \mathbf{q}^{14} \mathbf{t}^{10}\left(1+\mathbf{q}^{8} \mathbf{t}^{6}\right)\left(2+\mathbf{q}^{4} \mathbf{t}+\mathbf{q}^{6} \mathbf{t}^{3}\right)}{1-\mathbf{q}^{6} \mathbf{t}^{4}}+\right. \\
& \left.\left.+\frac{\left(1+\mathbf{q}^{4} \mathbf{t}\right) \cdot \mathbf{q}^{10} \mathbf{t}^{8}\left(1+\mathbf{q}^{2} \mathbf{t}\right)\left(\left(1-\mathbf{q}^{2} \mathbf{t}\right)\left(1+\mathbf{q}^{4} \mathbf{t}^{2}+\mathbf{q}^{6} \mathbf{t}^{4}+\mathbf{q}^{8} \mathbf{t}^{6}\right)+\mathbf{q}^{8} \mathbf{t}^{4}\left(1+\mathbf{q}^{2} \mathbf{t}^{2}\right)\right)}{1-\mathbf{q}^{6} \mathbf{t}^{4}}\right] \cdot \frac{1-\left(\mathbf{q}^{10} \mathbf{t}^{8}\right)^{\frac{n-4}{4}}}{1-\mathbf{q}^{10} \mathbf{t}^{8}}\right) \\
& \mathcal{K}_{u r}^{[5, n]}=\left(-\mathbf{q}^{2} \mathbf{t}\right)^{2(n-1)} \cdot\left([2]+\left(1+\mathbf{q}^{4} \mathbf{t}\right) \cdot\left(\mathbf{q}^{3} \mathbf{t}^{2}\left(1+\mathbf{q}^{8} \mathbf{t}^{\mathbf{6}}\right)+[2] \cdot \mathbf{q}^{\mathbf{6}} \mathbf{t}^{4}\left(1+\mathbf{q}^{2} \mathbf{t}^{2}\right)\right)-\right. \\
& -\left(1+\mathbf{q}^{4} \mathbf{t}\right) \cdot \frac{\mathbf{q}^{19} \mathbf{t}^{13}\left(1+\mathbf{q}^{8} \mathbf{t}^{\mathbf{6}}\right) \cdot\left(\mathbf{t} \cdot\left(1+\mathbf{q}^{4} \mathbf{t}+\mathbf{q}^{6} \mathbf{t}^{3}+\mathbf{q}^{6} \mathbf{t}^{4}\right)+[2] \mathbf{q} \cdot\left(1+\mathbf{q}^{2} \mathbf{t}^{3}\right)\right)}{1-\mathbf{q}^{\mathbf{6}} \mathbf{t}^{4}} \frac{1-\left(\mathbf{q}^{\mathbf{1 6}} \mathbf{t}^{12}\right)^{\frac{n-4}{4}}}{1-\mathbf{q}^{16} \mathbf{t}^{12}}+
\end{aligned}
$$




$$
\begin{aligned}
& +\left(1+\mathbf{q}^{4} \mathbf{t}\right) \cdot\left[\frac{\mathbf{q}^{13} \mathbf{t}^{10}\left(1+\mathbf{q}^{8} \mathbf{t}^{6}\right)\left(2+\mathbf{q}^{4} \mathbf{t}+\mathbf{q}^{6} \mathbf{t}^{3}\right)}{1-\mathbf{q}^{6} \mathbf{t}^{4}}+\right. \\
& \left.\left.+\frac{[2] \cdot \mathbf{q}^{10} \mathbf{t}^{8}\left(1+\mathbf{q}^{2} \mathbf{t}\right)\left(\left(1-\mathbf{q}^{2} \mathbf{t}\right)\left(1+\mathbf{q}^{4} \mathbf{t}^{2}+\mathbf{q}^{6} \mathbf{t}^{4}+\mathbf{q}^{8} \mathbf{t}^{6}\right)+\mathbf{q}^{8} \mathbf{t}^{4}\left(1+\mathbf{q}^{2} \mathbf{t}^{2}\right)\right)}{1-\mathbf{q}^{6} \mathbf{t}^{4}}\right] \cdot \mathbf{q}^{10} \mathbf{t}^{8} \cdot \frac{1-\left(\mathbf{q}^{10} \mathbf{t}^{8}\right)^{\frac{n-4}{4}}}{1-\mathbf{q}^{10} \mathbf{t}^{8}}\right)
\end{aligned}
$$

Some of these formulas could be considerably simplified, as shown in a couple of considered examples, but we put them in the form with explicit separation of $\left(1+\mathbf{q}^{2} \mathbf{t}\right)$ and $\left(1+\mathbf{q}^{4} \mathbf{t}\right)$ structures, when relation between reduced and unreduced polynomials is provided by the rule (4.18). It is this structure that should be revealed in order to understand the $N$ reduction of the superpolynomials [72].

One can easily check the $[m, n] \longleftrightarrow[n, m]$ equivalence:

$$
\mathcal{K}^{[5,2]}=\mathcal{K}^{[2,5]}, \quad \mathcal{K}^{[5,3]}=\mathcal{K}^{[3,5]}, \quad \mathcal{K}^{[5,4]}=\mathcal{K}^{[4,5]},
$$

while $n \longrightarrow-n$ relation fails:

$$
\mathcal{K}^{[5,-n]}(\mathbf{q}, \mathbf{t}) \nsucc \mathcal{K}^{[5, n]}\left(\mathbf{q}^{-1}, \mathbf{t}^{-1}\right)
$$

moreover, the l.h.s. is not a positive polynomial.

\section{Projective limit}

If one throws away all the $n$-power factors from the formulas for $\mathcal{K}$, i.e., keep only the contribution of the Young diagram $Q_{0}=[\mathrm{m}]$, one gets the projective limit of Khovanov polynomials, studied in [41], which are positive series. Explicit expressions are easily reproduced from our general formulas:

$$
\begin{aligned}
& { }_{r} K_{\square}^{[2, \infty]}=1+\frac{\mathbf{q}^{4} \mathbf{t}^{2}}{1-\mathbf{q}^{2} \mathbf{t}}=\frac{1+\mathbf{q}^{6} \mathbf{t}^{3}}{1-\mathbf{q}^{4} \mathbf{t}^{2}}=1+\left(1+\mathbf{q}^{2} \mathbf{t}\right) \cdot \frac{\mathbf{q}^{4} \mathbf{t}^{2}}{1-\mathbf{q}^{4} \mathbf{t}^{2}} \\
& { }_{u r} K_{\square}^{[2, \infty]}=\mathbf{q}+\frac{1}{\mathbf{q}} \cdot \frac{1+\mathbf{q}^{8} \mathbf{t}^{3}}{1-\mathbf{q}^{4} \mathbf{t}^{2}}=[2]+\left(1+\mathbf{q}^{4} \mathbf{t}\right) \cdot \frac{\mathbf{q}^{3} \mathbf{t}^{2}}{1-\mathbf{q}^{4} \mathbf{t}^{2}} \\
& { }_{r} K_{\square, \infty]}^{[3, \infty]}=\frac{\left(1+\mathbf{q}^{4} \mathbf{t}^{2}\right)\left(1+\mathbf{q}^{6} \mathbf{t}^{3}\right)}{1-\mathbf{q}^{6} \mathbf{t}^{4}}=1+\left(1+\mathbf{q}^{2} \mathbf{t}\right) \cdot \frac{\mathbf{q}^{4} \mathbf{t}^{2}}{1-\mathbf{q}^{6} \mathbf{t}^{4}}+\left(1+\mathbf{q}^{4} \mathbf{t}\right) \cdot \frac{\mathbf{q}^{6} \mathbf{t}^{4}}{1-\mathbf{q}^{6} \mathbf{t}^{4}} \\
& { }_{u r} K_{\square}^{[3, \infty]}=\frac{1}{\mathbf{q}} \cdot \frac{1+\mathbf{q}^{2}+\mathbf{q}^{4} \mathbf{t}^{2}+\mathbf{q}^{8} \mathbf{t}^{3}+\mathbf{q}^{10} \mathbf{t}^{5}+\mathbf{q}^{12} \mathbf{t}^{5}}{1-\mathbf{q}^{6} \mathbf{t}^{4}}=[2]+\left(1+\mathbf{q}^{4} \mathbf{t}\right) \cdot \frac{\mathbf{q}^{3} \mathbf{t}^{2}}{1-\mathbf{q}^{6} \mathbf{t}^{4}}+[2] \cdot\left(1+\mathbf{q}^{4} \mathbf{t}\right) \cdot \frac{\mathbf{q}^{6} \mathbf{t}^{4}}{1-\mathbf{q}^{6} \mathbf{t}^{4}} \\
& { }_{r} K_{\square}^{[4, \infty]}=1+\left(1+\mathbf{q}^{2} \mathbf{t}\right) \cdot \frac{\mathbf{q}^{4} \mathbf{t}^{2}\left(1+\mathbf{q}^{8} \mathbf{t}^{6}\right)}{1-\mathbf{q}^{8} \mathbf{t}^{6}}+\left(1+\mathbf{q}^{4} \mathbf{t}\right) \cdot \frac{\mathbf{q}^{6} \mathbf{t}^{4}\left(1+\mathbf{q}^{2} \mathbf{t}^{2}\right)}{1-\mathbf{q}^{8} \mathbf{t}^{6}}+\left(1+\mathbf{q}^{2} \mathbf{t}\right) \cdot \frac{\mathbf{q}^{18} \mathbf{t}^{11}(1+\mathbf{t})}{\left(1-\mathbf{q}^{6} \mathbf{t}^{4}\right)\left(1-\mathbf{q}^{8} \mathbf{t}^{6}\right)} \\
& { }_{u r} K_{\square}^{[4, \infty]}=[2]+\left(1+\mathbf{q}^{4} \mathbf{t}\right) \cdot \frac{\mathbf{q}^{3} \mathbf{t}^{2}\left(1+\mathbf{q}^{8} \mathbf{t}^{6}\right)}{1-\mathbf{q}^{8} \mathbf{t}^{6}}+[2] \cdot\left(1+\mathbf{q}^{4} \mathbf{t}\right) \cdot \frac{\mathbf{q}^{6} \mathbf{t}^{4}\left(1+\mathbf{q}^{2} \mathbf{t}^{2}\right)}{1-\mathbf{q}^{8} \mathbf{t}^{6}}+\left(1+\mathbf{q}^{4} \mathbf{t}\right) \cdot \frac{\mathbf{q}^{17} \mathbf{t}^{11}(1+\mathbf{t})}{\left(1-\mathbf{q}^{6} \mathbf{t}^{4}\right)\left(1-\mathbf{q}^{8} \mathbf{t}^{6}\right)} \\
& { }_{r} K_{\square}^{[5, \infty]}=1+\left(1+\mathbf{q}^{2} \mathbf{t}\right) \cdot \frac{\mathbf{q}^{4} \mathbf{t}^{2}}{1-\mathbf{q}^{10} \mathbf{t}^{8}} \cdot\left(1+\mathbf{q}^{8} \mathbf{t}^{6}+\frac{\mathbf{q}^{10} \mathbf{t}^{8}\left(1+\mathbf{q}^{4} \mathbf{t}\right)}{1-\mathbf{q}^{8} \mathbf{t}^{6}}\right)+ \\
& +\left(1+\mathbf{q}^{4} \mathbf{t}\right) \cdot \frac{\mathbf{q}^{6} \mathbf{t}^{4}}{1-\mathbf{q}^{10} \mathbf{t}^{8}} \cdot\left(1+\mathbf{q}^{2} \mathbf{t}^{2}+\mathbf{q}^{4} \mathbf{t}^{4}+\frac{\mathbf{q}^{10} \mathbf{t}^{8}\left(1+\mathbf{q}^{6} \mathbf{t}^{3}\right)}{1-\mathbf{q}^{8} \mathbf{t}^{6}}\right)+\left(1+\mathbf{q}^{2} \mathbf{t}\right) \cdot \frac{\mathbf{q}^{20} \mathbf{t}^{13}(1+\mathbf{t})}{\left(1-\mathbf{q}^{8} \mathbf{t}^{6}\right)\left(1-\mathbf{q}^{10} \mathbf{t}^{8}\right)} \\
& { }_{u r} K_{\square}^{[5, \infty]}=[2]+\left(1+\mathbf{q}^{4} \mathbf{t}\right) \cdot \frac{\mathbf{q}^{3} \mathbf{t}^{2}}{1-\mathbf{q}^{10} \mathbf{t}^{8}} \cdot\left(1+\mathbf{q}^{8} \mathbf{t}^{6}+\frac{\mathbf{q}^{10} \mathbf{t}^{8}\left(1+\mathbf{q}^{4} \mathbf{t}\right)}{1-\mathbf{q}^{8} \mathbf{t}^{6}}\right)+ \\
& +[2] \cdot\left(1+\mathbf{q}^{4} \mathbf{t}\right) \cdot \frac{\mathbf{q}^{6} \mathbf{t}^{4}}{1-\mathbf{q}^{10} \mathbf{t}^{8}} \cdot\left(1+\mathbf{q}^{2} \mathbf{t}^{2}+\mathbf{q}^{4} \mathbf{t}^{4}+\frac{\mathbf{q}^{10} \mathbf{t}^{8}\left(1+\mathbf{q}^{6} \mathbf{t}^{3}\right)}{1-\mathbf{q}^{8} \mathbf{t}^{6}}\right)+\left(1+\mathbf{q}^{4} \mathbf{t}\right) \cdot \frac{\mathbf{q}^{19} \mathbf{t}^{13}(1+\mathbf{t})}{\left(1-\mathbf{q}^{8} \mathbf{t}^{6}\right)\left(1-\mathbf{q}^{10} \mathbf{t}^{8}\right)}
\end{aligned}
$$


The difference between branches disappears in this limit. Unreduced polynomials are obtained from reduced ones by multiplication by [2] and application of the rule (4.18): $[2] \cdot\left(1+\mathbf{q}^{2} \mathbf{t}\right) \cong \frac{1}{\mathbf{q}} \cdot\left(1+\mathbf{q}^{4} \mathbf{t}\right)$. Despite $K_{\square}^{[m, \infty]}$ are nothing but Khovanov's substitutes of the nicely factorized MacDonald dimensions $M_{[m]}^{*}$ at $N=2$, they fail to fully factorize, starting already from $m=4$ :

$$
\begin{aligned}
& { }_{r} K_{\square}^{[2, \infty]}=\frac{1+\mathbf{q}^{\mathbf{6}} \mathbf{t}^{\mathbf{3}}}{1-\mathbf{q}^{\mathbf{4}} \mathbf{t}^{\mathbf{2}}} \\
& { }_{r} K_{\square}^{[3, \infty]}=\frac{\left(1+\mathbf{q}^{6} \mathbf{t}^{3}\right)}{1-\mathbf{q}^{6} \mathbf{t}^{4}} \cdot\left(1+\mathbf{q}^{4} \mathbf{t}^{2}\right) \\
& { }_{r} K_{\square}^{[4, \infty]}=\frac{1+\mathbf{q}^{6} \mathbf{t}^{3}}{\left(1-\mathbf{q}^{6} \mathbf{t}^{4}\right)\left(1-\mathbf{q}^{\mathbf{8}} \mathbf{t}^{\mathbf{6}}\right)} \cdot\left(1+\mathbf{q}^{4} \mathbf{t}^{2}\left(1-\mathbf{q}^{6} \mathbf{t}^{4}\right)+\mathbf{q}^{14} \mathbf{t}^{\mathbf{9}}\right) \\
& { }_{r} K_{\square}^{[5, \infty]}=\frac{1+\mathbf{q}^{6} \mathbf{t}^{3}}{\left(1-\mathbf{q}^{8} \mathbf{t}^{6}\right)\left(1-\mathbf{q}^{10} \mathbf{t}^{8}\right)} \cdot\left(1+\mathbf{q}^{4} \mathbf{t}^{2}+\mathbf{q}^{6} \mathbf{t}^{4}+\mathbf{q}^{14} \mathbf{t}^{9}+\mathbf{q}^{16} \mathbf{t}^{11}+\mathbf{q}^{20} \mathbf{t}^{13}\right) \\
& { }_{r} K_{\square}^{[6, \infty]}=\frac{1+\mathbf{q}^{6} \mathbf{t}^{3}}{\left(1-\mathbf{q}^{\mathbf{8}} \mathbf{t}^{\mathbf{6}}\right)\left(1-\mathbf{q}^{\mathbf{1 0}} \mathbf{t}^{\mathbf{8}}\right)\left(1-\mathbf{q}^{\mathbf{1 2}} \mathbf{t}^{\mathbf{1 0}}\right)} . \\
& \cdot\left(1+\left(\mathbf{q}^{4} \mathbf{t}^{2}+\mathbf{q}^{14} \mathbf{t}^{9}\right)\left(1-\mathbf{q}^{16} \mathbf{t}^{12}\right)+\left(\mathbf{q}^{6} \mathbf{t}^{4}+\mathbf{q}^{20} \mathbf{t}^{13}\right)\left(1-\mathbf{q}^{8} \mathbf{t}^{6}\right)+\mathbf{q}^{16} \mathbf{t}^{11}+\mathbf{q}^{18} \mathbf{t}^{13}+\mathbf{q}^{34} \mathbf{t}^{24}\right) \\
& \text {... }
\end{aligned}
$$

Formulas are written in the form, where positivity of the series is explicitly seen.

\section{Recursion for KR polynomials at $N=3$ and $m=4$}

KR polynomial with $N=3$ coincides with the superpolynomial at $m=2,3$, and deviates from it for $m \geq 4$. For $m=4$ four out of five terms in the evolution formula will contribute, and the four $n$-independent coefficients can be found from the known expressions for particular polynomials computed with [44]. For reduced case we get for $n=1 \bmod 4$ :

$$
\begin{aligned}
& { }_{r} \mathcal{K}_{\square}^{[4, n]}(N=3)=\left(-\mathbf{q}^{4} \mathbf{t}\right)^{\frac{3(n-1)}{2}} \cdot\left(1+\frac{\mathbf{q}^{4} \mathbf{t}^{2}\left(1+\mathbf{q}^{8} \mathbf{t}^{3}\right)}{\left(1-\mathbf{q}^{4} \mathbf{t}^{2}\right)\left(1-\mathbf{q}^{8} \mathbf{t}^{4}\right)} \cdot\left(1+\mathbf{q}^{2} \mathbf{t}^{2}+\mathbf{q}^{4} \mathbf{t}+\mathbf{q}^{4} \mathbf{t}^{4}+\mathbf{q}^{6} \mathbf{t}^{3}+\mathbf{q}^{6} \mathbf{t}^{4}-\right.\right. \\
& \left.-\mathbf{q}^{8} \mathbf{t}^{4}-\mathbf{q}^{10} \mathbf{t}^{4}+\mathbf{q}^{10} \mathbf{t}^{5}-\mathbf{q}^{12} \mathbf{t}^{6}-\mathbf{q}^{14} \mathbf{t}^{5}-\mathbf{q}^{14} \mathbf{t}^{8}-\mathbf{q}^{16} \mathbf{t}^{7}+\mathbf{q}^{16} \mathbf{t}^{9}-\mathbf{q}^{18} \mathbf{t}^{9}-\mathbf{q}^{20} \mathbf{t}^{9}\right) \cdot \frac{1-\left(\mathbf{q}^{8} \mathbf{t}^{6}\right)^{\frac{n-1}{4}}}{1-\mathbf{q}^{8} \mathbf{t}^{6}}+ \\
& +\frac{\left(1-\mathbf{t}^{2}\right) \cdot \mathbf{q}^{\mathbf{1 0}} \mathbf{t}^{4}\left(1+\mathbf{q}^{4} \mathbf{t}\right)\left(1+\mathbf{q}^{8} \mathbf{t}^{\mathbf{3}}\right)}{\left(1-\mathbf{q}^{4} \mathbf{t}^{2}\right)^{2}} \cdot \frac{1-\left(\mathbf{q}^{\mathbf{1 2}} \mathbf{t}^{\mathbf{8}}\right)^{\frac{n-1}{4}}}{1-\mathbf{q}^{6} \mathbf{t}^{4}} \\
& -\underbrace{\mathbf{q}^{\mathbf{1 0}} \mathbf{t}^{4}\left(1+\mathbf{q}^{4} \mathbf{t}\right)\left(1+\mathbf{q}^{2} \mathbf{t}+\mathbf{q}^{2} \mathbf{t}^{2}+\mathbf{q}^{8} \mathbf{t}^{3}\right)}_{\mathbf{q}^{10} \mathbf{t}^{4}\left(1+\mathbf{q}^{4} \mathbf{t}\right)\left(\left(1+\mathbf{q}^{2} \mathbf{t}\right)+\mathbf{q}^{2} \mathbf{t}^{2}\left(1+\mathbf{q}^{6} \mathbf{t}\right)\right)} \cdot \frac{1-\left(\mathbf{q}^{16} \mathbf{t}^{10}\right)^{\frac{n-1}{4}}}{\left(1-\mathbf{q}^{4} \mathbf{t}^{2}\right)\left(1-\mathbf{q}^{\mathbf{8}} \mathbf{t}^{4}\right)})
\end{aligned}
$$

and

$$
\begin{aligned}
& { }_{u r} \mathcal{K}_{\square}^{[4, n]}(N=3)=\left(-\mathbf{q}^{4} \mathbf{t}\right)^{\frac{3(n-1)}{2}} \cdot\left([3]+\frac{\mathbf{q}^{2} \mathbf{t}^{2}\left(1+\mathbf{q}^{6} \mathbf{t}\right)}{\left(1-\mathbf{q}^{4} \mathbf{t}^{2}\right)\left(1-\mathbf{q}^{8} \mathbf{t}^{4}\right)}\right. \\
& \cdot\left(1+\mathbf{q}^{2}+\mathbf{q}^{2} \mathbf{t}^{2}+\mathbf{q}^{4} \mathbf{t}^{2}+\mathbf{q}^{4} \mathbf{t}^{4}+2 \mathbf{q}^{6} \mathbf{t}^{4}+\mathbf{q}^{8} \mathbf{t}^{3}+\mathbf{q}^{8} \mathbf{t}^{4}+\mathbf{q}^{10} \mathbf{t}^{3}--2 \mathbf{q}^{10} \mathbf{t}^{4}+2 \mathbf{q}^{12} \mathbf{t}^{5}-2 \mathbf{q}^{12} \mathbf{t}^{6}-\mathbf{q}^{14} \mathbf{t}^{6}+\right. \\
& \left.+2 \mathbf{q}^{14} \mathbf{t}^{7}-\mathbf{q}^{14} \mathbf{t}^{8}+\mathbf{q}^{16} \mathbf{t}^{7}-2 \mathbf{q}^{16} \mathbf{t}^{8}-\mathbf{q}^{18} \mathbf{t}^{7}+\mathbf{q}^{18} \mathbf{t}^{9}-\mathbf{q}^{20} \mathbf{t}^{7}-\mathbf{q}^{20} \mathbf{t}^{9}-\mathbf{q}^{22} \mathbf{t}^{9}-\mathbf{q}^{22} \mathbf{t}^{11}-2 \mathbf{q}^{24} \mathbf{t}^{11}-\mathbf{q}^{26} \mathbf{t}^{11}\right)
\end{aligned}
$$




$$
\begin{aligned}
& \cdot \frac{1-\left(\mathbf{q}^{8} \mathbf{t}^{6}\right)^{\frac{n-1}{4}}}{1-\mathbf{q}^{8} \mathbf{t}^{\mathbf{6}}}++\left(1+\mathbf{q}^{6} \mathbf{t}\right) \cdot \frac{(1+\mathbf{t}) \cdot \mathbf{q}^{8} \mathbf{t}^{4}}{\left(1-\mathbf{q}^{4} \mathbf{t}^{2}\right)^{2}} \cdot\left(1-\mathbf{t}-\mathbf{q}^{2} \mathbf{t}+\mathbf{q}^{8} \mathbf{t}^{3}+\mathbf{q}^{10} \mathbf{t}^{3}-\mathbf{q}^{10} \mathbf{t}^{4}\right) \cdot \frac{\left(1-\mathbf{q}^{12} \mathbf{t}^{8}\right)^{\frac{n-1}{4}}}{1-\mathbf{q}^{6} \mathbf{t}^{4}}- \\
& -\underbrace{\mathbf{q}^{8} \mathbf{t}^{4}\left(1+\mathbf{q}^{6} \mathbf{t}\right)\left(1+\mathbf{q}^{2} \mathbf{t}^{2}+\mathbf{q}^{4} \mathbf{t}+\mathbf{q}^{4} \mathbf{t}^{2}+\mathbf{q}^{8} \mathbf{t}^{3}+\mathbf{q}^{10} \mathbf{t}^{3}\right)}_{\mathbf{q}^{10} \mathbf{t}^{4}\left(1+\mathbf{q}^{6} \mathbf{t}\right)\left(\frac{1}{\mathbf{q}^{2}}\left(1+\mathbf{q}^{4} \mathbf{t}\right)+\frac{1}{\mathbf{q}}[2] \cdot \mathbf{q}^{2} \mathbf{t}^{2}\left(1+\mathbf{q}^{6} \mathbf{t}\right)\right.} \cdot \frac{1-\left(\mathbf{q}^{16} \mathbf{t}^{10}\right)^{\frac{n-1}{4}}}{\left(1-\mathbf{q}^{4} \mathbf{t}^{2}\right)\left(1-\mathbf{q}^{8} \mathbf{t}^{4}\right)})
\end{aligned}
$$

while for $n=-1 \bmod 4$

$$
\begin{aligned}
& { }_{r} \mathcal{K}_{\square}^{[4, n]}(N=3)=\left(-\mathbf{q}^{4} \mathbf{t}\right)^{\frac{3(n-1)}{2}} \cdot\left(1+\mathbf{q}^{4} \mathbf{t}^{2} \cdot\left(1+\mathbf{q}^{4} \mathbf{t}\right)\left(1+\mathbf{q}^{2} \mathbf{t}^{2}+\mathbf{q}^{4} \mathbf{t}^{2}+\mathbf{q}^{8} \mathbf{t}^{4}+\mathbf{q}^{10} \mathbf{t}^{5}\right)+\frac{\mathbf{q}^{8} \mathbf{t}^{6}\left(1+\mathbf{q}^{8} \mathbf{t}^{3}\right)}{\left(1-\mathbf{q}^{4} \mathbf{t}^{2}\right)\left(1-\mathbf{q}^{8} \mathbf{t}^{4}\right)}\right. \\
& \cdot\left(1+\mathbf{q}^{2}-\mathbf{q}^{4}+\mathbf{q}^{4} \mathbf{t}^{2}+\mathbf{q}^{6} \mathbf{t}+\mathbf{q}^{6} \mathbf{t}^{4}+\mathbf{q}^{8} \mathbf{t}^{3}-\mathbf{q}^{10} \mathbf{t}^{4}+\mathbf{q}^{10} \mathbf{t}^{\mathbf{5}}\right. \\
& \left.+\mathbf{q}^{12} \mathbf{t}^{5}-\mathbf{q}^{14} \mathbf{t}^{\mathbf{5}}-\mathbf{q}^{14} \mathbf{t}^{6}-\mathbf{q}^{16} \mathbf{t}^{5}-\mathbf{q}^{16} \mathbf{t}^{8}-\mathbf{q}^{18} \mathbf{t}^{7}-\mathbf{q}^{\mathbf{2 0}} \mathbf{t}^{9}\right) \cdot \frac{1-\left(\mathbf{q}^{8} \mathbf{t}^{6}\right)^{\frac{n-3}{4}}}{1-\mathbf{q}^{8} \mathbf{t}^{6}}+ \\
& +\frac{\left(1-\mathbf{t}^{2}\right) \cdot \mathbf{q}^{16} \mathbf{t}^{8}\left(1+\mathbf{q}^{4} \mathbf{t}\right)\left(1+\mathbf{q}^{8} \mathbf{t}^{3}\right)}{\left(1-\mathbf{q}^{4} \mathbf{t}^{2}\right)^{2}} \cdot \frac{1-\left(\mathbf{q}^{12} \mathbf{t}^{8}\right)^{\frac{n-3}{4}}}{1-\mathbf{q}^{6} \mathbf{t}^{4}} \\
& -\underbrace{\mathbf{q}^{18} \mathbf{t}^{10}\left(1+\mathbf{q}^{4} \mathbf{t}\right)\left(1+\mathbf{q}^{6} \mathbf{t}+\mathbf{q}^{6} \mathbf{t}^{2}+\mathbf{q}^{8} \mathbf{t}^{3}\right)}_{\mathbf{q}^{18} \mathbf{t}^{10}\left(\left(1+\mathbf{q}^{4} \mathbf{t}\right)\left(1+\mathbf{q}^{6} \mathbf{t}\right)+\mathbf{q}^{6} \mathbf{t}^{2}\left(1+\mathbf{q}^{2} \mathbf{t}\right)\left(1+\mathbf{q}^{4} \mathbf{t}\right)\right.} \cdot \frac{1-\left(\mathbf{q}^{16} \mathbf{t}^{10}\right)^{\frac{n-3}{4}}}{\left(1-\mathbf{q}^{4} \mathbf{t}^{2}\right)\left(1-\mathbf{q}^{8} \mathbf{t}^{4}\right)})
\end{aligned}
$$

and

$$
\begin{aligned}
& { }_{u r} \mathcal{K}_{\square}^{[4, n]}(N=3)=\left(-\mathbf{q}^{4} \mathbf{t}\right)^{\frac{3(n-1)}{2}} \\
& \cdot\left([3]+\left(1+\mathbf{q}^{6} \mathbf{t}\right) \cdot \mathbf{q}^{2} \mathbf{t}^{2}\left(1+\mathbf{q}^{2}+[2]^{2} \mathbf{q}^{4} \mathbf{t}^{2}+\mathbf{q}^{8} \mathbf{t}^{4}+\mathbf{q}^{12} \mathbf{t}^{5}\right)+\frac{\mathbf{q}^{8} \mathbf{t}^{6}\left(1+\mathbf{q}^{6} \mathbf{t}\right)}{\left(1-\mathbf{q}^{4} \mathbf{t}^{2}\right)\left(1-\mathbf{q}^{8} \mathbf{t}^{4}\right)}\right. \\
& \cdot\left([3]++1+\mathbf{q}^{2} \mathbf{t}^{2}+\mathbf{q}^{4} \mathbf{t}^{2}+\mathbf{q}^{4} \mathbf{t}^{4}-\mathbf{q}^{6} \mathbf{t}^{2}+\mathbf{q}^{6} \mathbf{t}^{4}+2 \mathbf{q}^{8} \mathbf{t}^{3}-\mathbf{q}^{8} \mathbf{t}^{4}+\mathbf{q}^{10} \mathbf{t}^{3}-2 \mathbf{q}^{10} \mathbf{t}^{4}+\mathbf{q}^{10} \mathbf{t}^{5}+2 \mathbf{q}^{12} \mathbf{t}^{5}-2 \mathbf{q}^{12} \mathbf{t}^{6}+\right. \\
& \left.+2 \mathbf{q}^{14} \mathbf{t}^{7}-\mathbf{q}^{14} \mathbf{t}^{8}-\mathbf{q}^{16} \mathbf{t}^{7}-\mathbf{q}^{16} \mathbf{t}^{8}-2 \mathbf{q}^{18} \mathbf{t}^{7}-\mathbf{q}^{20} \mathbf{t}^{7}-\mathbf{q}^{20} \mathbf{t}^{9}-\mathbf{q}^{22} \mathbf{t}^{9}-\mathbf{q}^{22} \mathbf{t}^{11}-\mathbf{q}^{24} \mathbf{t}^{11}\right) \cdot \frac{1-\left(\mathbf{q}^{8} \mathbf{t}^{6}\right)^{\frac{n-3}{4}}}{1-\mathbf{q}^{8} \mathbf{t}^{6}}+ \\
& +\left(1+\mathbf{q}^{6} \mathbf{t}\right) \cdot \frac{(1+\mathbf{t}) \cdot \mathbf{q}^{14} \mathbf{t}^{8}}{\left(1-\mathbf{q}^{4} \mathbf{t}^{2}\right)^{2}} \cdot\left(1-\mathbf{t}-\mathbf{q}^{2} \mathbf{t}+\mathbf{q}^{8} \mathbf{t}^{3}+\mathbf{q}^{10} \mathbf{t}^{3}-\mathbf{q}^{10} \mathbf{t}^{4}\right) \cdot \frac{\left(1-\mathbf{q}^{12} \mathbf{t}^{8}\right)^{\frac{n-3}{4}}}{1-\mathbf{q}^{6} \mathbf{t}^{4}}- \\
& -\underbrace{(1+\mathbf{t})\left(1+\mathbf{q}^{2}+\mathbf{q}^{6} \mathbf{t}+\mathbf{q}^{6} \mathbf{t}^{2}+\mathbf{q}^{8} \mathbf{t}+\mathbf{q}^{10} \mathbf{t}^{3}\right)}_{\mathbf{q}^{18} \mathbf{t}^{10}\left(1+\mathbf{q}^{6} \mathbf{t}\right)\left(\frac{1}{\mathbf{q}} \mathbf{t}^{10}\left(1+\left(1+\mathbf{q}^{6} \mathbf{t}\right)+\frac{1}{\mathbf{q}^{2}} \mathbf{q}^{6} \mathbf{t}^{2}\left(1+\mathbf{q}^{4} \mathbf{t}\right)\right)\right.} \cdot \frac{1-\left(\mathbf{q}^{16} \mathbf{t}^{10}\right)^{\frac{n-3}{4}}}{\left(1-\mathbf{q}^{4} \mathbf{t}^{2}\right)\left(1-\mathbf{q}^{8} \mathbf{t}^{4}\right)})
\end{aligned}
$$

Throwing away all the $n$-dependent powers we obtain the projective limit:

$$
\begin{aligned}
& { }_{r} \mathcal{K}_{\square}^{[4, \infty]}(N=3)=\frac{\left(1+\mathbf{q}^{\mathbf{8}} \mathbf{t}^{\mathbf{3}}\right)\left(1+\mathbf{q}^{\mathbf{1 0}} \mathbf{t}^{\mathbf{5}}\right)}{\left(1-\mathbf{q}^{\mathbf{6}} \mathbf{t}^{\mathbf{4}}\right)\left(1-\mathbf{q}^{\mathbf{8}} \mathbf{t}^{\mathbf{6}}\right)}\left(1+\mathbf{q}^{\mathbf{4}} \mathbf{t}^{\mathbf{2}}+\mathbf{q}^{\mathbf{8}} \mathbf{t}^{4}\right)=1+\frac{\mathbf{q}^{\mathbf{4}} \mathbf{t}^{\mathbf{2}}}{\left(1-\mathbf{q}^{\mathbf{6}} \mathbf{t}^{\mathbf{4}}\right)\left(1-\mathbf{q}^{\mathbf{8}} \mathbf{t}^{\mathbf{6}}\right)} \\
& \cdot\left(\left(1+\mathbf{q}^{\mathbf{2}} \mathbf{t}\right) \cdot \mathbf{q}^{\mathbf{1 2}} \mathbf{t}^{\mathbf{5}}+\left(1+\mathbf{q}^{\mathbf{4}} \mathbf{t}\right)\left(1+[2] \mathbf{q}^{\mathbf{3}} \mathbf{t}^{\mathbf{2}}+\mathbf{q}^{\mathbf{1 4}} \mathbf{t}^{\mathbf{7}}\right)+\left(1+\mathbf{q}^{\mathbf{6}} \mathbf{t}\right) \cdot\left(\mathbf{q}^{\mathbf{4}} \mathbf{t}^{\mathbf{4}}\left(1-\mathbf{q}^{\mathbf{6}} \mathbf{t}^{\mathbf{4}}\right)+\mathbf{q}^{\mathbf{1 6}} \mathbf{t}^{\mathbf{9}}\right)\right)
\end{aligned}
$$

in full accordance with $[42,43]$. Since the expression in the first line is proportional to $\left(1+\mathbf{q}^{2} \mathbf{t}\right)$, in unreduced case one could expect

$$
? \frac{1}{\mathbf{q}^{2}} \frac{\left(1+\mathbf{q}^{8} \mathbf{t}^{3}\right)\left(1+\mathbf{q}^{6} \mathbf{t}\right)}{\left(1-\mathbf{q}^{6} \mathbf{t}^{4}\right)\left(1-\mathbf{q}^{8} \mathbf{t}^{6}\right)}\left(1+\mathbf{q}^{4} \mathbf{t}^{2}+\mathbf{q}^{8} \mathbf{t}^{4}\right) \cdot \frac{1+\mathbf{q}^{\mathbf{1 0}} \mathbf{t}^{\mathbf{5}}}{1+\mathbf{q}^{\mathbf{2}} \mathbf{t}} ?
$$


However, this is not a positive series. The true unreduced expression arises by application of the rules

$$
[3]\left(1+\mathbf{q}^{2} \mathbf{t}\right) \longrightarrow \frac{1}{\mathbf{q}^{\mathbf{2}}}\left(1+\mathbf{q}^{\mathbf{6}} \mathbf{t}\right), \quad \swarrow^{[3][2] \mathbf{q}\left(1+\mathbf{q}^{\mathbf{6}} \mathbf{t}\right)} \searrow
$$

to decomposition in the second line:

$$
\begin{aligned}
& { }_{u r} \mathcal{K}_{\square}^{[4, \infty]}(N=3) \\
& =[3]+\frac{\mathbf{q}^{\mathbf{2}} \mathbf{t}^{\mathbf{2}}\left(1+\mathbf{q}^{\mathbf{6}} \mathbf{t}\right)\left(1+\mathbf{q}^{2}+[2]^{2} \mathbf{q}^{\mathbf{4}} \mathbf{t}^{\mathbf{2}}+[3] \mathbf{q}^{\mathbf{6}} \mathbf{t}^{\mathbf{4}}\left(1-\mathbf{q}^{\mathbf{6}} \mathbf{t}^{\mathbf{4}}\right)+\mathbf{q}^{\mathbf{1 2}} \mathbf{t}^{\mathbf{5}}+[2] \mathbf{q}^{\mathbf{1 5}} \mathbf{t}^{\mathbf{7}}+[3] \mathbf{q}^{\mathbf{1 8}} \mathbf{t}^{\mathbf{9}}\right)}{\left(1-\mathbf{q}^{\mathbf{6}} \mathbf{t}^{\mathbf{4}}\right)\left(1-\mathbf{q}^{\mathbf{8}} \mathbf{t}^{\mathbf{6}}\right)}
\end{aligned}
$$

The same rules convert above expressions for the full reduced polynomials $\mathcal{K}^{[4, n]}$ into unreduced ones - this is illustrated by expansions of particular coefficients, not captured by projective limit. In the product of two (or more) differentials reduction acts on the lowest

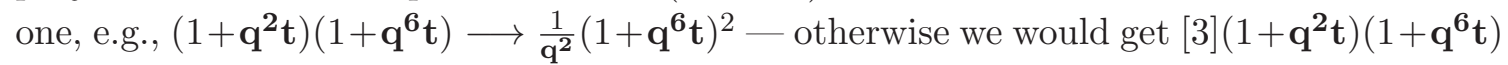
which contains three time more items.

Topological invariance implies that

$$
\mathcal{K}_{r}^{[4,3]}(N=3)=\mathcal{K}_{r}^{[3,4]}(N=3)=\mathcal{P}_{r}^{[3,4]}\left(\mathbf{a}=\mathbf{q}^{3}\right)
$$

what is indeed the case. For unreduced polynomials the analogue of the second relation is incorrect, because unreduced KR polynomial always differs from unreduced superpolynomial, even for $N=m=3$, see [72].

\section{Remnant of differential expansion}

Differential expansions (DE) of [57] and [58-60] play an increasingly important role in modern theory of knot polynomials. Essential for KR reductions will be a complementary nested DE [72]. Somewhat amusingly, the ordinary DE partly survives the Khovanov reduction at $N=2$ as well. Namely, while

$$
\begin{aligned}
& \mathcal{P}_{\square}^{[m, n]}-1 \vdots\{A q\}\{A / t\} \sim\left(\mathbf{a}^{2} \mathbf{q}^{2} \mathbf{t}^{\mathbf{3}}\right) \cdot\left(1+\frac{\mathbf{a}^{2} \mathbf{t}}{\mathbf{q}^{\mathbf{2}}}\right) \Longrightarrow \\
& \Longrightarrow \mathcal{P}_{\square}^{[m, n]}\left(\mathbf{a}=\mathbf{q}^{\mathbf{2}}\right)-1 \vdots\left(1+\mathbf{q}^{\mathbf{2}} \mathbf{t}\right)\left(1+\mathbf{q}^{\mathbf{6}} \mathbf{t}^{\mathbf{3}}\right) \sim \Longrightarrow \mathcal{P}_{\square}^{[m, n]}\left(\mathbf{a}=\mathbf{q}^{\mathbf{2}}\right)-1 \vdots\left(1+\mathbf{q}^{\mathbf{2}} \mathbf{t}\right)^{2}
\end{aligned}
$$

for Khovanov polynomials we have:

$$
\mathbf{q}^{\gamma_{[m, n]}} \cdot \mathcal{K}_{\square}^{[m, n]}-1 \vdots\left(1+\mathbf{q}^{2} \mathbf{t}\right)
$$

but the power at the r.h.s. can not be raised from one to two.

We emphasize that the normalization of Khovanov polynomials is already fixed by the condition (3.8),

$$
\mathcal{K}_{\square}^{[m, n]}(N=2)-\mathcal{P}_{\square}^{[m, n]}\left(\mathbf{a}=\mathbf{q}^{\mathbf{2}}\right) \vdots(1+\mathbf{t})
$$


thus the mysterious power of $\mathbf{q}$ at the l.h.s. should have some objective meaning. It actually depends on the number of strands $m$ and, as usual, on the residue $r=n \bmod m$ :

\begin{tabular}{|c|c|c|c|c|c|c|c|c|c|c|c|c|c|}
\hline$m$ & 2 & 3 & & 4 & & 5 & & & & 6 & & $\ldots$ & $m$ \\
\hline$n$ & $1+2 k$ & $1+3 k$ & $2+3 k$ & $1+4 k$ & $3+4 k$ & $1+5 k$ & $2+5 k$ & $3+5 k$ & $4+5 k$ & $1+6 k$ & $5+6 k$ & $1+m k$ \\
\hline$\gamma_{[m, n]}$ & 0 & $2 k$ & $2 k$ & $4 k$ & $2+4 k$ & $8 k$ & $8 k$ & $2+8 k$ & $4+8 k$ & $12 k$ & $8+12 k$ & $2 k \cdot \operatorname{entier}\left(\frac{(m-1)^{2}}{4}\right)$ \\
\hline
\end{tabular}

The generating function for the coefficients in front of $k$ is $\sum_{m} \operatorname{entier}\left(\frac{(m-1)^{2}}{4}\right) \cdot x^{m}=$ $\frac{x^{2}}{(1-x)^{2}\left(1-x^{2}\right)}$, residue-dependence is easily restored from the topological identity $\gamma_{[m, r]}=$ $\gamma_{[r, m]}$. Differential $d_{1} \sim\{A / t\},{ }^{2}$ which is surviving in these formulas in the form $\left(1+\mathbf{q}^{2} \mathbf{t}\right)$, is responsible for the $\mathrm{U}(1)$-reduction of knot polynomials, i.e. the DE relation (10.2) implies that there is some memory about the $\mathrm{U}(1)$ reduction, surviving when that to $\mathrm{U}(2)$ is performed, but there is a nontrivial correction when $\mathcal{K}$ is not just $\mathcal{P}\left(\mathbf{a}=\mathbf{q}^{\mathbf{2}}\right)$, i.e. when $\xi \neq 0$ in (3.10).

For KR polynomials at $N=3$ - the only beyond $N=2$ one available at the moment - the structure is more complicated

$$
\mathbf{q}^{?} \cdot \mathcal{K}_{\square}^{[m, n]}(N=3)-1 \vdots\left(1+\mathbf{q}^{\mathbf{4}} \mathbf{t}\right)
$$

for any power of q. Still some structure clearly exists in this case as well - and deserves closer attention.

\section{Conclusion}

In this paper we made six claims:

(a) KR polynomials for torus knots possess just the same $n$-evolution expansion as the superpolynomials:

$$
K_{R}^{[m, n]}(N, \mathbf{q}, \mathbf{t})=\sum_{Q \in R^{\otimes m}} k_{Q}(N, \mathbf{q}, \mathbf{t}) \cdot \lambda_{Q}^{n}
$$

(b) When quantum dimension $D_{Q}$ vanishes, so does the expansion coefficient $k_{Q}$ :

$$
D_{Q}(N)=0 \Longrightarrow M_{Q}^{*}(N) \Longrightarrow k_{Q}(N)=0
$$

and we get a shortened recursion, which needs less initial conditions.

(c) In variance with (1.2) for reduced torus superpolynomials, the evolution expansion (11.1) for their KR counterparts is not consistent with the mirror symmetry under $n \longleftrightarrow-n$. Strictly speaking, the lack of covariance under $n \longleftrightarrow-n$ is not a problem, because the corresponding knot-diagrams are not Reidemeister equivalent. Still the two knots, though topologically different, are related by a mirror map and thus one could expect a relation like (1.6). Moreover, if we had a control over these transformation laws, the knowledge of any particular KR polynomial would provide two initial conditions. For $m=2,3$ this

\footnotetext{
${ }^{2}$ Strictly speaking, for $\mathbf{a}=\mathbf{q}^{2}$ the factor $\{A q\}$ is also proportional to $d_{1}$ — this is the origin of the square at the r.h.s. of (10.1) — but most probably this factor is fully destroyed by the Khovanov reduction.
} 
mirror anomaly is concentrated in the framing factor and thus is indeed comprehensible, however it becomes far more complicated for $m \geq 4$. Technically it is related to the presence of negative items in the coefficients in front of the fractions $\frac{1-\lambda^{n}}{1-\lambda}$. Actually these negative items are absorbed into structures, proportional to $(1-\lambda)$, and thus do not spoil positivity of entire polynomial - but this works only for $n>0$, while at $n<0$ such combinations fail to be sign-definite and can not pretend to be super- and KR polynomials. This is an unpleasant property of the evolution formulas, already encountered in a similar situation in [61].

(d) The knowledge of the evolution recursion in $n$ allows one to easily calculate Khovanov polynomials with $N=2$ for arbitrary torus knots, beginning actually from the unknot(!): one does it first for arbitrary $n$ at $m=2$ and then proceeds recursively in $m$, using the topological identity between $[m, n]$ and $[n, m]$. Important here is the claim (b): dramatic reduction of recursion degree, which at $N=2$ grows linearly with $m$, what makes above identity sufficient for the $m$-recursion

(e) Though differential expansion [58-60], implying that

$$
\mathcal{P}_{\square}-1 \sim\{A q\}\{A / t\}
$$

for fundamental HOMFLY and superpolynomials in topological framing, is violated for KR polynomials, it is substituted by a weaker, still powerful structure: a linear decomposition of properly normalized $K_{\square}$ into differentials:

$$
K_{\square}(N)-1=\oplus_{k=1}^{N}\left(1+\mathbf{q}^{2 k} \mathbf{t}\right)
$$

As a simplest application, reduction rule (4.4) applies nicely to Khovanov polynomials and it allows to directly obtain unreduced polynomials from reduced ones. In result unreduced Khovanov polynomial always satisfies

$$
{ }_{u r} K_{\square}(2)-[2] \sim\left(1+\mathbf{q}^{\mathbf{4}} \mathbf{t}\right)
$$

However, this kind of rule are not sufficient to further deduce reduced Khovanov per se from superpolynomials and more effort is needed for this purpose. More relevant can be the nested structure, which helps to fix the ambiguities in linear decomposition, - but this story is beyond the scope of this letter.

(f) A more traditional version of differential expansion is found to survive for Khovanov polynomials $(N=2)$ in a somewhat surprising form of $(10.2)$, with additional powers of q, specific for torus knots. It would be interesting to see what happens to these relations beyond the present scope - of torus knot and fundamental representations.

\section{Acknowledgments}

We are indebted to Eugene Gorsky for discussions, encouragement and sharing his lists of torus KR polynomials for $N=2$ and $N=3$. We also appreciate the comments of Dror Bar-Natan and Lukas Lewark. We are grateful to Gleb Aminov, Semeon Arthamonov, and Alexander Popolitov for help with computing Khovanov polynomials and to Alexander Shumakovitch for sharing his program for reduced Khovanov homologies.

This work was supported by the Russian Science Foundation (Grant No.16-12-10344). 
Open Access. This article is distributed under the terms of the Creative Commons Attribution License (CC-BY 4.0), which permits any use, distribution and reproduction in any medium, provided the original author(s) and source are credited.

\section{References}

[1] J.W. Alexander, Topological invariants of knots and links, Trans. Amer. Math. Soc. 30 (1928) 275.

[2] V.F.R. Jones, Index for subfactors, Invent. Math. 72 (1983) 1.

[3] V.F.R. Jones, A polynomial invariant for knots via von Neumann algebras, Bull. Amer. Math. Soc. 12 (1985) 103.

[4] V.F.R. Jones, Hecke algebra representations of braid groups and link polynomials, Annals Math. 126 (1987) 335 [inSPIRE].

[5] L.H. Kauffman, State models and the Jones polynomial, Topology 26 (1987) 395.

[6] P. Freyd et al., A new polynomial invariant of knots and links, Bull. Amer. Math. Soc. 12 (1985) 239.

[7] J.H. Przytycki and K.P. Traczyk, Invariants of links of Conway type, Kobe J. Math. 4 (1987) 115 [arXiv: 1610.06679].

[8] A. Yu. Morozov, Are there p-adic knot invariants?, Theor. Math. Phys. 187 (2016) 447 [Teor. Mat. Fiz. 187 (2016) 3] [arXiv: 1509. 04928] [INSPIRE].

[9] S.-S. Chern and J. Simons, Some cohomology classes in principal fiber bundles and their application to Riemannian geometry, Proc. Nat. Acad. Sci. 68 (1971) 791.

[10] S.-S. Chern and J. Simons, Characteristic forms and geometric invariants, Annals Math. 99 (1974) 48 [INSPIRE].

[11] A.S. Schwarz, New topological invariants in the theory of quantized fields, in Baku Topol. Conf., (1987).

[12] E. Witten, Quantum field theory and the Jones polynomial, Commun. Math. Phys. 121 (1989) 351 [INSPIRE].

[13] M. Atiyah, The geometry and physics of knots, Cambridge University Press, Cambridge U.K., (1990).

[14] M. Aganagic and S. Shakirov, Knot homology and refined Chern-Simons index, Commun. Math. Phys. 333 (2015) 187 [arXiv:1105.5117] [InSPIRE].

[15] M. Aganagic and S. Shakirov, Refined Chern-Simons theory and knot homology, Proc. Symp. Pure Math. 85 (2012) 3 [arXiv:1202 .2489] [INSPIRE].

[16] M. Aganagic and S. Shakirov, Refined Chern-Simons theory and topological string, arXiv: 1210.2733 [INSPIRE].

[17] N. Yu. Reshetikhin and V.G. Turaev, Ribbon graphs and their invariants derived from quantum groups, Commun. Math. Phys. 127 (1990) 1 [INSPIRE].

[18] E. Guadagnini, M. Martellini and M. Mintchev, Chern-Simons field theory and quantum groups, in Clausthal Procs., (1989), pg. 307. 
[19] E. Guadagnini, M. Martellini and M. Mintchev, Chern-Simons holonomies and the appearance of quantum groups, Phys. Lett. B 235 (1990) 275 [INSPIRE].

[20] P. Rama Devi, T.R. Govindarajan and R.K. Kaul, Three-dimensional Chern-Simons theory as a theory of knots and links. 3. Compact semisimple group, Nucl. Phys. B 402 (1993) 548 [hep-th/9212110] [INSPIRE].

[21] P. Ramadevi, T.R. Govindarajan and R.K. Kaul, Knot invariants from rational conformal field theories, Nucl. Phys. B 422 (1994) 291 [hep-th/9312215] [INSPIRE].

[22] P. Ramadevi, T.R. Govindarajan and R.K. Kaul, Representations of composite braids and invariants for mutant knots and links in Chern-Simons field theories,

Mod. Phys. Lett. A 10 (1995) 1635 [hep-th/9412084] [INSPIRE].

[23] P. Ramadevi, T.R. Govindarajan and R.K. Kaul, Chirality of knots $9_{42}$ and $10_{71}$ and Chern-Simons theory, Mod. Phys. Lett. A 9 (1994) 3205 [hep-th/9401095] [InSPIRE].

[24] A. Morozov and A. Smirnov, Chern-Simons theory in the temporal gauge and knot invariants through the universal quantum R-matrix, Nucl. Phys. B 835 (2010) 284 [arXiv:1001.2003] [INSPIRE].

[25] A. Anokhina, On R-matrix approaches to knot invariants, arXiv:1412.8444 [INSPIRE].

[26] A. Mironov, A. Morozov and An. Morozov, Character expansion for HOMFLY polynomials. I. Integrability and difference equations, arXiv:1112.5754.

[27] A. Mironov, A. Morozov and A. Morozov, Character expansion for HOMFLY polynomials. II. Fundamental representation. Up to five strands in braid, JHEP 03 (2012) 034 [arXiv:1112.2654] [INSPIRE].

[28] A. Anokhina, A. Mironov, A. Morozov and A. Morozov, Colored HOMFLY polynomials as multiple sums over paths or standard Young tableaux, Adv. High Energy Phys. 2013 (2013) 931830 [arXiv: 1304.1486] [INSPIRE].

[29] A. Anokhina and A. Morozov, Cabling procedure for the colored HOMFLY polynomials, Theor. Math. Phys. 178 (2014) 1 [Teor. Mat. Fiz. 178 (2014) 3] [arXiv:1307.2216] [INSPIRE].

[30] M. Khovanov, A categorification of the Jones polynomial, Duke Math. J. 101 (2000) 359 [math/9908171] [INSPIRE].

[31] M. Khovanov and L. Rozansky, Matrix factorizations and link homology, Fund. Math. 199 (2008) 1.

[32] D. Bar-Natan, On Khovanov's categorification of the Jones polynomial, Algebr. Geom. Topol. 2 (2002) 337 [math.QA/0201043].

[33] The Knot atlas webpage, http://www.katlas.org.

[34] S. Gukov, A.S. Schwarz and C. Vafa, Khovanov-Rozansky homology and topological strings, Lett. Math. Phys. 74 (2005) 53 [hep-th/0412243] [INSPIRE].

[35] N.M. Dunfield, S. Gukov and J. Rasmussen, The superpolynomial for knot homologies, Experiment. Math. 15 (2006) 129 [math.GT/0505662] [INSPIRE].

[36] E. Gorsky, S. Gukov and M. Stosic, Quadruply-graded colored homology of knots, arXiv:1304.3481 [INSPIRE].

[37] V. Dolotin and A. Morozov, Introduction to Khovanov homologies. I. Unreduced Jones superpolynomial, JHEP 01 (2013) 065 [arXiv: 1208.4994] [INSPIRE]. 
[38] V. Dolotin and A. Morozov, Introduction to Khovanov homologies. II. Reduced Jones superpolynomials, J. Phys. Conf. Ser. 411 (2013) 012013 [arXiv: 1209.5109] [InSPIRE].

[39] V. Dolotin and A. Morozov, Introduction to Khovanov homologies. III. A new and simple tensor-algebra construction of Khovanov-Rozansky invariants, Nucl. Phys. B 878 (2014) 12 [arXiv: 1308.5759] [INSPIRE].

[40] A. Anokhina and A. Morozov, Towards R-matrix construction of Khovanov-Rozansky polynomials. I. Primary T-deformation of HOMFLY, JHEP 07 (2014) 063 [arXiv: 1403.8087] [INSPIRE].

[41] E. Gorsky, A. Oblomkov and J. Rasmussen, On stable Khovanov homology of torus knots, Experiment. Math. 22 (2013) 265 [arXiv:1206.2226].

[42] L. Lewark, sl-foam homology calculations, Algebr. Geom. Topol. 13 (2013) 3661 [arXiv: 1212.2553] [INSPIRE].

[43] E. Gorsky and L. Lewark, On stable sl-homology of torus knots, Experiment. Math. 24 (2015) 162 [arXiv:1404.0623].

[44] Foamho, an sl3-homology calculator webpage, http://lewark.de/lukas/foamho.html.

[45] S. Nawata and A. Oblomkov, Lectures on knot homology, Contemp. Math. 680 (2016) 137 [arXiv: 1510.01795] [INSPIRE].

[46] D. Galakhov and G.W. Moore, Comments on the two-dimensional Landau-Ginzburg approach to link homology, arXiv:1607.04222 [INSPIRE].

[47] D. Galakhov, Why is Landau-Ginzburg link cohomology equivalent to Khovanov homology?, arXiv: 1702.07086 [INSPIRE].

[48] A. Anokhina, Towards formalization of the soliton counting technique for the Khovanov-Rozansky invariants in the deformed R-matrix approach, arXiv:1710.07306 [INSPIRE].

[49] A.A. Belavin, A.M. Polyakov and A.B. Zamolodchikov, Infinite conformal symmetry in two-dimensional quantum field theory, Nucl. Phys. B 241 (1984) 333 [INSPIRE].

[50] A. Zamolodchikov and Al. Zamolodchikov, Conformal field theory and critical phenomena in $2 d$ systems, (2009).

[51] V.S. Dotsenko and V.A. Fateev, Conformal algebra and multipoint correlation functions in two-dimensional statistical models, Nucl. Phys. B 240 (1984) 312 [INSPIRE].

[52] A. Gerasimov, A. Morozov, M. Olshanetsky, A. Marshakov and S.L. Shatashvili, Wess-Zumino-Witten model as a theory of free fields, Int. J. Mod. Phys. A 5 (1990) 2495 [INSPIRE].

[53] L. Alvarez-Gaume, Random surfaces, statistical mechanics and string theory, Helv. Phys. Acta 64 (1991) 359 [InSPIRE].

[54] P. Di Francesco, P. Mathieu and D. Sènèchal, Conformal field theory, Springer, New York U.S.A., (1997) [INSPIRE].

[55] A. Mironov, S. Mironov, A. Morozov and A. Morozov, CFT exercises for the needs of AGT, arXiv:0908.2064 [INSPIRE].

[56] P. Dunin-Barkowski, A. Mironov, A. Morozov, A. Sleptsov and A. Smirnov, Superpolynomials for toric knots from evolution induced by cut-and-join operators, JHEP 03 (2013) 021 [arXiv:1106.4305] [INSPIRE]. 
[57] A. Mironov, A. Morozov and A. Morozov, Evolution method and "differential hierarchy" of colored knot polynomials, AIP Conf. Proc. 1562 (2013) 123 [arXiv:1306.3197] [InSPIRE].

[58] H. Itoyama, A. Mironov, A. Morozov and A. Morozov, HOMFLY and superpolynomials for figure eight knot in all symmetric and antisymmetric representations, JHEP 07 (2012) 131 [arXiv: 1203.5978] [INSPIRE].

[59] S.B. Arthamonov, A. Mironov and A. Morozov, Differential hierarchy and additional grading of knot polynomials, Theor. Math. Phys. 179 (2014) 509 [Teor. Mat. Fiz. 179 (2014) 147] [arXiv: 1306.5682] [INSPIRE].

[60] Ya. Kononov and A. Morozov, On the defect and stability of differential expansion, JETP Lett. 101 (2015) 831 [Pisma Zh. Eksp. Teor. Fiz. 101 (2015) 931] [arXiv: 1504.07146] [INSPIRE].

[61] A. Morozov, Knot polynomials for twist satellites, arXiv:1801.02407 [INSPIRE].

[62] M. Rosso and V.F.R. Jones, On the invariants of torus knots derived from quantum groups, J. Knot Theor. Ramificat. 02 (1993) 97.

[63] X.-S. Lin and H. Zheng, On the Hecke algebras and the colored HOMFLY polynomial, Trans. Amer. Math. Soc. 362 (2010) 1 [math. QA/0601267].

[64] M. Tierz, Soft matrix models and Chern-Simons partition functions, Mod. Phys. Lett. A 19 (2004) 1365 [hep-th/0212128] [INSPIRE].

[65] A. Brini, B. Eynard and M. Mariño, Torus knots and mirror symmetry, Annales Henri Poincaré 13 (2012) 1873 [arXiv:1105.2012] [INSPIRE].

[66] A. Aleksandrov, A.D. Mironov, A. Morozov and A.A. Morozov, Towards matrix model representation of HOMFLY polynomials, JETP Lett. 100 (2014) 271 [Pisma Zh. Eksp. Teor. Fiz. 100 (2014) 297] [arXiv: 1407.3754] [INSPIRE].

[67] I. Cherednik, Jones polynomials of torus knots via DAHA, arXiv:1111.6195 [INSPIRE].

[68] A. Mironov, A. Morozov, S. Shakirov and A. Sleptsov, Interplay between MacDonald and Hall-Littlewood expansions of extended torus superpolynomials, JHEP 05 (2012) 070 [arXiv: 1201.3339] [INSPIRE].

[69] A. Mironov, A. Morozov and S. Shakirov, Torus HOMFLY as the Hall-Littlewood polynomials, J. Phys. A 45 (2012) 355202 [arXiv:1203.0667] [INSPIRE].

[70] S. Shakirov, Colored knot amplitudes and Hall-Littlewood polynomials, arXiv:1308.3838 [INSPIRE].

[71] E. Gorsky, private communication.

[72] A. Anokhina and A. Morozov, Nested differential expansion and reductions to small $N$ in the simplified Khovanov-Rozansky calculus, in preparation. 